Article type :Original Article

5

6

7

8

\title{
Monitoring Plasmodium falciparum and Plasmodium vivax using microsatellite markers indicates limited changes in population structure after substantial transmission decline in Papua New Guinea
}

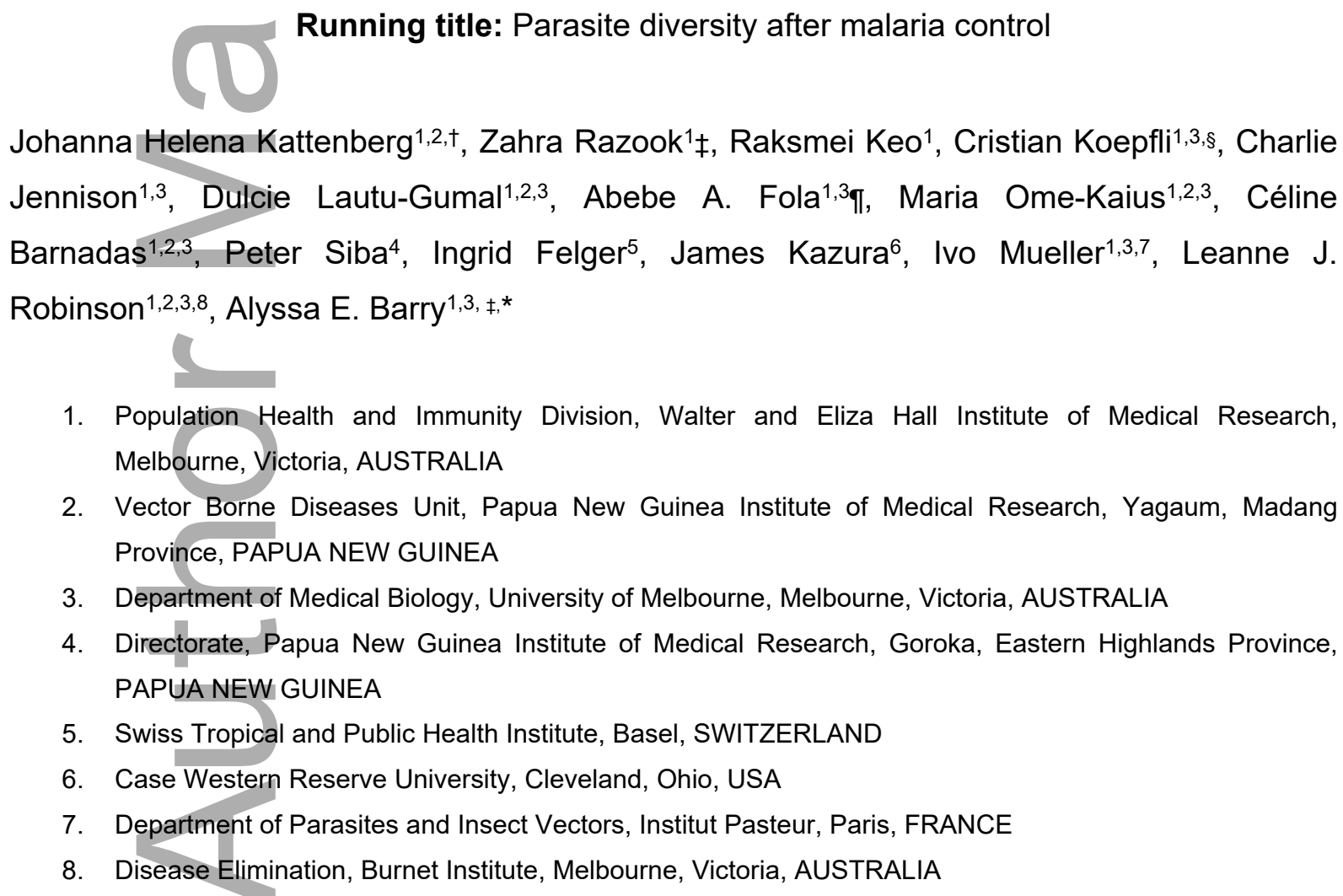
Jennison ${ }^{1,3}$, Dulcie Lautu-Gumal ${ }^{1,2,3}$, Abebe A. Fola ${ }^{1,3} \pi$, Maria Ome-Kaius ${ }^{1,2,3}$, Céline Barnadas $^{1,2,3}$, Peter Siba ${ }^{4}$, Ingrid Felger $^{5}$, James Kazura ${ }^{6}$, Ivo Mueller ${ }^{1,3,7}$, Leanne J. Robinson ${ }^{1,2,3,8}$, Alyssa E. Barry ${ }^{1,3, \ddagger, *}$

1. Population Health and Immunity Division, Walter and Eliza Hall Institute of Medical Research, Melbourne, Victoria, AUSTRALIA

2. Vector Borne Diseases Unit, Papua New Guinea Institute of Medical Research, Yagaum, Madang Province, PAPUA NEW GUINEA

3. Department of Medical Biology, University of Melbourne, Melbourne, Victoria, AUSTRALIA

4. Directorate, Papua New Guinea Institute of Medical Research, Goroka, Eastern Highlands Province, PAPUA NEW GUINEA

5. Swiss Tropical and Public Health Institute, Basel, SWITZERLAND

6. Case Western Reserve University, Cleveland, Ohio, USA

7. Department of Parasites and Insect Vectors, Institut Pasteur, Paris, FRANCE

8. Disease Elimination, Burnet Institute, Melbourne, Victoria, AUSTRALIA

† Current affiliation: Department of Biomedical Sciences, Institute of Tropical Medicine, Antwerp, BELGIUM

This is the author manuscript accepted for publication and has undergone full peer review but has not been through the copyediting, typesetting, pagination and proofreading process, which may lead to differences between this version and the Version of Record. Please cite this article as doi: 10.1111/MEC.15654

This article is protected by copyright. All rights reserved 
$\ddagger$ Current affiliation: School of Medicine, Deakin University, Geelong, AUSTRALIA and Burnet Institute, Melbourne, Victoria, AUSTRALIA

$\S$ Current affiliation: Department of Biological Sciences, University of Notre Dame, Notre Dame, Indiana, USA I Current affiliation: Department of Biological Sciences, Purdue University, West Lafayette, Indiana, USA

\section{*Corresponding Author:}

School of Medicine, Deakin University

Nicol Drive North, Waurn Ponds,

Victoria, AUSTRALIA

Phone: +61352278777

Email: a.barry@deakin.edu.au

\section{ABSTRACT}

Monitoring the genetic structure of pathogen populations may be an economical and sensitive approach to quantify the impact of control on transmission dynamics, highlighting the need for a better understanding of changes in population genetic parameters as transmission declines. Here we describe the first population genetic analysis of the major human malaria parasites, Plasmodium falciparum (Pf) and Plasmodium vivax (Pv) following nationwide distribution of long-lasting insecticide treated nets (LLIN) in Papua New Guinea (PNG). Parasite isolates from pre- (2005-6) and post-LLIN (2010-2014) were genotyped using microsatellite markers. Despite parasite prevalence declining substantially (East Sepik Province: $P f=54.9-8.5 \%, P v=35.7-5.6 \%$, Madang Province: $P f=38.0-9.0 \%, P v: 31.8-19.7 \%$ ), genetically diverse and intermixing parasite populations remained. Pf diversity declined modestly post-LLIN relative to pre-LLIN (East Sepik: $R_{\mathrm{s}}=7.1-6.4, H_{\mathrm{e}}=0.77-0.71$; Madang: $\left.R_{\mathrm{s}}=8.2-6.1, H_{\mathrm{e}}=0.79-0.71\right)$. Unexpectedly, population structure present in pre-LLIN populations was lost post-LLIN, suggesting that more frequent human movement between provinces may have contributed to higher gene flow. $P v$ prevalence initially declined but increased again in one province, yet diversity remained high throughout the study period (East Sepik: $R_{\mathrm{s}}=11.4-9.3, H_{\mathrm{e}}=0.83-0.80$; Madang: $R_{\mathrm{s}}=12.2-14.5, H_{\mathrm{e}}=0.85-0.88$ ). Although genetic differentiation values increased between provinces over time, no significant population structure was observed at any time point. For both species, a decline in multiple infections and increasing clonal transmission and significant multilocus linkage disequilibrium $(\mathrm{mLD})$ post-LLIN was a positive indicator of impact on the parasite population using microsatellite markers. These parameters may be useful adjuncts to traditional epidemiological tools in the early stages of transmission reduction.

\section{Keywords}

This article is protected by copyright. All rights reserved 
Plasmodium falciparum, Plasmodium vivax, malaria control, transmission dynamics, population genetics, microsatellite markers, Papua New Guinea

\section{INTRODUCTION}

Characterising pathogen transmission dynamics using population genomics is essential to guide containment efforts and to plan strategies for disease elimination (Grad \& Lipsitch, 2014; Hedtke et al., 2019; Wlodarska, Johnston, Gardy, \& Tang, 2015). Pathogen populations comprise genetically distinct individuals that are related to varying degrees due to the accumulation of genetic variation as they transmit from host to host. Genomic diversity within populations can thereby indicate the extent of transmission intensity, whilst that between populations determines their connectivity (gene flow) and is influenced by local selection and inbreeding. Measuring pairwise relationships between infections further identifies how infections are spreading from host to host within a population and allows epidemiological characteristics of transmission to be defined (e.g. endemic versus epidemic). Understanding how these population genetic parameters change under the pressure of control interventions is central to using genomic epidemiology as an effective tool to monitor pathogen transmission dynamics.

When utilising population genetics to measure transmission dynamics it is important to consider how genomic diversity is generated. Human malaria parasites acquire de novo mutations whilst replicating asexually and reassortment occurs through sexual recombination within the mosquito vector. However, the generation of novel recombinants occurs only if the mosquito has taken up multiple, genetically distinct clones in the blood meal, otherwise selffertilization occurs, and progeny are clonal. Outcrossing is therefore dependent on the presence of multiple genetically distinct infections in the human host and increases with endemicity (Babiker et al., 1994; Paul et al., 1995). The population structure of the most virulent malaria parasite, Plasmodium falciparum is associated with transmission intensity (Anderson et al., 2000). At moderate to high transmission where multiclonal infections are frequently found, parasite populations are characterised by high diversity and a lack of population structure with low levels of linkage disequilibrium (LD) (Anderson et al., 2000; Gatei et al., 2010; Orjuela-Sanchez et al., 2013; Salgueiro, Vicente, Figueiredo, \& Pinto, 2016; Schultz et al., 2010). At low transmission where multiclonal infections are less common, clonal transmission and inbreeding amongst closely related individuals is more common, resulting in lower overall diversity and high levels of $L D$, whilst population structure is more evident due to both lower gene flow between areas and within population transmission dynamics (Anderson et al., 2000; Branch et al., 2011; Chenet, Schneider, Villegas, \& Escalante, 2012; Noviyanti et al., 2015). For P. vivax, also a significant human 
pathogen, relapsing infections and other unique features that enhance its transmission (Olliaro et al., 2016), result in a higher prevalence of multiclonal infections. Therefore, $P$. vivax populations are often characterised by high genetic diversity, even at low transmission (Ferreira et al., 2007; Fola et al., 2017; Gunawardena, Ferreira, Kapilananda, Wirth, \& Karunaweera, 2014; Noviyanti et al., 2015; Waltmann et al., 2018). In the South West Pacific region, a modest decline in diversity and increasing population structure occurs with the eastward decline in transmission (Fola et al., 2017; Koepfli et al., 2013; Waltmann et al., 2018). LD and pockets of clonal $P$. vivax transmission have been observed in several studies, suggesting increasingly focal transmission as malaria rates decline (Abdullah et al., 2013; Batista, Barbosa, Da Silva Bastos, Viana, \& Ferreira, 2015; Chenet et al., 2012; Delgado-Ratto et al., 2016; Ferreira et al., 2007; Imwong et al., 2007; Iwagami et al., 2012; Noviyanti et al., 2015; Orjuela-Sanchez et al., 2013). Comparative analyses show P. vivax has a higher effective transmission intensity (Hofmann et al., 2017; Lin et al., 2010; Robinson et al., 2015) and higher diversity than $P$. falciparum due to a longer association with humans and fewer population bottlenecks (Chenet et al., 2012; Gilabert et al., 2018; Hupalo et al., 2016; Jennison et al., 2015; Liu et al., 2014; Loy et al., 2017; Neafsey et al., 2012; Noviyanti et al., 2015; Orjuela-Sanchez et al., 2013; Pava et al., 2017). P. vivax is more resilient to control efforts and thus may be less likely to show changes in parasite population structure than P. falciparum (Barry, Waltmann, Koepfli, Barnadas, \& Mueller, 2015; Cornejo \& Escalante, 2006; Feachem et al., 2010; Liu et al., 2014; Neafsey et al., 2012; OliveiraFerreira et al., 2010; Waltmann et al., 2015). No studies have yet investigated the impact of intensified control on the population genetics of sympatric $P$. vivax and $P$. falciparum populations.

The worldwide scale up of malaria control since the early 2000s, has reduced transmission dramatically around the world. Indeed, between 2010 and 2016, disease incidence declined by $18 \%$ and mortality by $32 \%$ (WHO, 2017, 2019). The incidence of clinical cases and infection prevalence remain the mainstay of malaria surveillance however population genetic surveillance has emerged as a promising and high-resolution approach for malaria monitoring (Arnott, Barry, \& Reeder, 2012; Barry et al., 2015; Dalmat, Naughton, Kwan-Gett, Slyker, \& Stuckey, 2019; Koepfli \& Mueller, 2017; malEra Consultative Group on Monitoring \& Surveillance, 2011). Specifically, these approaches identify local transmission dynamics (e.g. endemic, epidemic, imported infections), connectivity between parasite populations in different endemic areas (Anderson et al., 2000; Fola et al., 2017; Noviyanti et al., 2015; Vardo-Zalik et al., 2013; Waltmann et al., 2018) and "sources and sinks", which together could help to design targeted control interventions (Auburn \& Barry, 2017; Barry et al., 2015; Koepfli \& Mueller, 2017). Population genetic surveys could also identify local drivers 
contributing to sustained transmission such as particular human social and economic interactions (Barry et al., 2015; Delgado-Ratto et al., 2016; Koepfli \& Mueller, 2017). While parasite population genetics and genomics is becoming more popular and accessible, the impact on control programs has been limited, and to date few studies have systematically assessed the long-term impact of malaria control using these approaches (Bei et al., 2018; Chenet, Taylor, Blair, Zuluaga, \& Escalante, 2015; R. F. Daniels et al., 2015; Gatei et al., 2010; Gunawardena et al., 2014; Nkhoma et al., 2013; Vardo-Zalik et al., 2013). Moreover, it is not clear how long transmission needs to be disrupted, or to which extent prevalence should be reduced, before changes in parasite population structure can be seen. A better understanding of the impact of malaria control interventions on $P$. falciparum and $P$. vivax population structure is urgently required to capitalise on the potential of genomic surveillance for malaria control and elimination.

Population genetic surveys using panels of well-validated neutral microsatellite markers (Anderson et al., 2000; Imwong et al., 2007; Karunaweera, Ferreira, Hartl, \& Wirth, 2006) were conducted on the north coast of Papua New Guinea before the intensification of malaria control (2005/2006). P. vivax showed higher genetic diversity and a lack of population structure yet there was significant population structure of $P$. falciparum populations (Jennison et al., 2015; Koepfli et al., 2013; Schultz et al., 2010; Waltmann et al., 2018). Significant inbreeding ( $m L D)$ was not observed for sub-populations of either species, confirming high levels of outcrossing and endemic transmission (Jennison et al., 2015). Since that time, PNG has implemented an intensified control program including the free nationwide distribution of Long Lasting Insecticide Treated Nets (LLIN). This resulted in a significant decline in infections across the country including the north coast provinces previously covered in our population genetic surveys (Arnott et al., 2013; Barry et al., 2013; Hetzel et al., 2016; Kattenberg et al., 2020; Koepfli et al., 2017; Koepfli et al., 2015). The impact on parasite population structure and transmission dynamics after the rollout of LLINs, however, remains unresolved. We sought to characterise the impact of reduced prevalence on the population structure of sympatric $P$. falciparum and $P$. vivax populations. Microsatellite haplotypes were generated from $P$. falciparum and $P$. vivax samples collected in multiple cross sectional surveys from 2010-14 after two rounds of mass LLIN distribution and compared to published data from isolates collected before the intensified malaria control program (Jennison et al., 2015; Schultz et al., 2010). The results show the impact of declining prevalence on PNG parasite populations and identify the critical parameters for monitoring these changes using microsatellite markers. 


\section{Study sites and design}

175

176

177

178

179

180

181

182

183

184

185

186

187

188

189

190

191

192

193

194

195

196

197

198

199

200

201

202

203

204

205

206

The studies were conducted in two Provinces on the highly endemic north coast region of Papua New Guinea (PNG) (Figure 1). In the WHO Western Pacific Region (WPR), the malaria mortality rate declined by $58 \%$ over the period 2010-2015, however infection prevalence in Papua New Guinea (PNG) remains the highest in this region (and outside the African continent), contributing $81 \%$ of malaria cases and $86 \%$ of malaria deaths in 2017 in the region $(\mathrm{WHO}, 2017,2018)$ primarily due to $P$. falciparum and $P$. vivax infections (Kattenberg et al., 2020; Koepfli et al., 2017; WHO, 2018). In 2003, a new national malaria control campaign was launched to achieve high levels of LLIN ownership and usage in PNG (Hetzel et al., 2014; Hetzel et al., 2012). Coverage with LLIN was low in most parts of the country before nationwide free distribution took place (2004-8 and 2009-2012) (Betuela et al., 2012; Genton et al., 1994; Hii et al., 2001). This resulted in a significant increase in ownership of bed nets across the country by 2010 (any type $80 \%$; LLINs 65\%) (Hetzel et al., 2014; Hetzel et al., 2012) and the average malaria incidence rate in sentinel sites dropped from $13 / 1,000$ population to $2 / 1,000$ (range $0.6-3.3 / 1000$ post-LLIN) (Hetzel et al., 2016).

On the hyperendemic north coast, $P$. falciparum PCR prevalence dropped from $38 \%$ to $12 \%$ and $P$. vivax prevalence decreased from $28 \%$ to $13 \%$ (Kattenberg et al., 2020; Koepfli et al., 2017) (Figure 1). Prevalence reductions were more substantial for $P$. falciparum than for $P$. vivax, as has been seen in many co-endemic areas (Feachem et al., 2010). In East Sepik Province, malaria decreased from a very high burden (73\% of surveyed individuals infected in 2005 as measured by molecular detection (LDR-FMA (Mueller et al., 2009)) to heterogeneous transmission (prevalence in villages ranging from $1 \%$ to $61 \%$, median $6 \%$, as measured by qPCR (Kattenberg et al., 2020)) after two rounds of LLIN distribution. An initial round of LLIN distribution was conducted between 2004 and 2009, followed by additional distributions in 2011/2012 and subsequently in 2014/15. In Madang province, however, malaria prevalence decreased from $63 \%$ to $28 \%$ by qPCR after the first round of LLIN distributions (Koepfli et al., 2017; Koepfli et al., 2015; Schultz et al., 2010). After the second LLIN distribution (2010-2014), P. falciparum continued to drop, however an increase in $P$. vivax prevalence was observed (from $13 \%$ to $20 \%$ by qPCR (Koepfli et al., 2017)). In Madang province, malaria prevalence was less heterogenous in the sampled villages than in East Sepik Province (Kattenberg et al., 2020; Koepfli et al., 2017; Koepfli et al., 2015).

Whole blood samples were collected from participants in cross-sectional studies conducted between 2005 and 2014 along the North Coast of PNG (Figure 1) (Arnott et al., 2013; 
Kattenberg et al., 2020; Koepfli et al., 2017; Koepfli et al., 2015; Mueller et al., 2009; Schultz et al., 2010). In Madang Province (MAD), the same three catchment areas were studied in 2006 (Schultz et al., 2010; Senn et al., 2012), 2010 (Koepfli et al., 2015) and 2014 (Koepfli et al., 2017). The study area included a selection of villages along a coastal stretch of $70 \mathrm{~km}$ (Mugil and Malala regions), surrounded by coconut and cocoa plantations and subsistence gardens, and one area approximately $50 \mathrm{~km}$ inland (Utu). Here, the climate is tropical with a rainy season from December to April. In East Sepik Province (ESP), participants in the Wosera Catchment (ESP1) including fourteen villages were sampled in 2005 during the dryseason (August-September) (Jennison et al., 2015; Senn et al., 2012). A broader survey (ESP2) was conducted in April-May 2005 including five catchment areas that were re-visited in 2012-13 (Kattenberg et al., 2020; Mueller et al., 2009). The study areas in ESP consist of an area of over $160 \mathrm{~km}^{2}$ with low hills and riverine plains with a wet, tropical climate (Genton et al., 1995). The natural vegetation is lowland hill forest that has mostly been replaced by regrowth following cultivation and wide grasslands on the plains near the Sepik River.

In all surveys, demographic and clinical information was collected, blood slides examined by expert microscopists and a blood sample collected in EDTA tubes for extraction of DNA. In the 2005 ESP studies, Plasmodium species were detected by Light Detection ReactionFluorescent Microspere Assay (LDR-FMA) (McNamara et al., 2006; Mueller et al., 2009), whereas in all other studies quantitative PCR (qPCR) detection by $\operatorname{TaqMan}^{\mathrm{TM}}$ assay was used (Rosanas-Urgell et al., 2010). To determine multiplicity of infection (MOI), P. falciparum positive samples were genotyped for $P f m s p 2$ and $P$. vivax positive samples were genotyped with Pvmsp1f3 and MS16 (ESP1 and MAD 2006) or Pvmsp1f3 and MS2 (MAD 2010 \& 2014 and ESP2 2005 \& ESP 2012-13) ), as previously described (Arnott et al., 2013; Kattenberg et al., 2020; Koepfli et al., 2017; Koepfli et al., 2015; Mueller et al., 2009; Schultz et al., 2010). The sample selection and genotyping procedures of the ESP1 2005 (Wosera) and MAD 2006 were as previously described (Arnott et al., 2013; Jennison et al., 2015; Schultz et al., 2010). For the other studies, samples with MOI of 1 were selected for further genotyping with the neutral microsatellite panels as described below. For the studies conducted after the large scale LLIN distribution (>2006) all monoclonal isolates (MOI=1) were included, but for the 2005 ESP2 population, a selection of samples was made for the analysis with the microsatellite panel (Table S1).

Written informed consent was obtained from all study participants or their parents or legal guardians. The study was approved by the PNG IMR Institutional Review Board (IRB\#11/16) and the PNG Medical Research Advisory Committee (MRAC 11/21), National Institutes of 
242 Health, Division of Microbiology and Infectious Diseases (DMID Protocol \#10-0035) and

243 Walter and Eliza Hall Institute Human Research Ethics Committee (HREC \#12/10).

\section{Genotyping procedures}

245 For both species, a panel of 9-10 neutral microsatellite markers were amplified in the selected samples (Table S1) using a multiplex primary PCR followed by individual nested

247 PCRs as previously described (Anderson et al., 2000; Jennison et al., 2015; Koepfli et al., 248 2013; Schultz et al., 2010). For $P$. falciparum, samples were genotyped at nine previously 249 validated and commonly used, putatively neutral, microsatellite loci including TA1, TAA60, 250 Polya, ARA2, Pfg377, TAA87, PfPK2, TAA81 and 2490 (Anderson et al., 2000; Schultz et al., 251 2010). For $P$. vivax, 10 putatively neutral microsatellites were genotyped as previously 252 described: MS1, MS2, MS5, MS6, MS7, MS9, MS10, MS12, MS15, and MS20 (Jennison et 253 al., 2015; Koepfli et al., 2013). All PCR products were sent to a commercial facility for 254 fragment analysis on an ABI3730xl platform (Applied Biosystems) using the size standard 255 LIZ500. Primers used were the same for all datasets (Jennison et al., 2015; Schultz et al., 256 2010).

\section{Analysis}

The electropherograms were analysed with Genemapper V4.0 (Applied Biosystems) with the same peak calling strategy as described previously (Jennison et al., 2015; Schultz et al., 2010). To avoid artefacts, precautions were taken to ensure allele calling was consistent (Jennison et al., 2015), and carefully reconstructing dominant haplotypes as per previously described methods (Anderson, Su, Bockarie, Lagog, \& Day, 1999; Jennison et al., 2015; Schultz et al., 2010). Briefly, this involved setting the minimum fluorescence to 500 Random Fluorescence Units (RFU) for all colours except the size standard. Stutter window was set to 3.5 for $3 \mathrm{bp}$ repeats and 4.5 for $4 \mathrm{bp}$ repeats. The stutter ratio was set to 0.4 for all markers. The stutter detection was only applied to shorter alleles, with longer alleles within the stutter window subject to the standard $30 \%$ cut-off threshold. Samples with low fluorescence were manually reanalysed with a minimum fluorescence of 100 RFU. For the Madang 2005 and Wosera 2006 P. falciparum data, previously published cleaned and rounded microsatellite allele repeat numbers for $P$. falciparum single clone infections (Schultz et al., 2010) were converted back to allele sizes using the known number of nucleotides/repeat, whereas for $P$. vivax the raw data (allele calls) was available (Jennison et al., 2015). These data were combined with the newly generated MS data from the other studies before binning the alleles using the TANDEM software (Matschiner \& Salzburger, 2009). Allele frequencies of the entire dataset (incl. previously genotyped datasets) were investigated and outlying alleles 
$278(60 \%)$ or more MS loci were excluded from further analysis. We attempted to calibrate the $P$. 279 falciparum data from pre-LLIN Madang 2006 and Wosera (ESP1 2005) by converting 280 rounded repeat numbers back to allele sizes, binning together with the newly generated data and removing outliers. However, there was strong population structure when compared to the new dataset, indicating experimental differences despite the use of the same protocols. Thus, we excluded direct comparisons between old and new datasets for $P$. falciparum.

To conduct the population genetic analyses, allele frequencies and input files for the various population genetics programs were created using CONVERT version 1.31 (Glaubitz) and PGD Spider version 2.1.0.1 (Lischer \& Excoffier, 2012). Genetic diversity was measured by calculating the number of alleles $(A)$, Nei's gene diversity (expected heterozygosity $(\mathrm{He})(\mathrm{Nei}$, 1987)) and allelic richness (Rs) (Hurlbert, 1971) that corrects for sample size, using FSTAT version 2.9.3.2 (Goudet, 1995). Pairwise genetic differentiation was measured by calculating pairwise Jost's $D$ (Jost, 2008) and Weir and Cockerhams $F_{\mathrm{ST}}$ (Weir \& Cockerham, 1984) values and $95 \%$ confidence intervals were estimated with 1000 bootstraps using the diveRsity package in R (Keenan, McGinnity, Cross, Crozier, \& Prodöhl, 2013). In contrast to some earlier studies (Schultz et al., 2010), where haploid genotypes were coded as diploid genotypes, but homozygote at each locus, the data in this study was analysed using haploid datasets (as in Jennison et al. (Jennison et al., 2015)). As a measure of inbreeding in each population, multilocus linkage disequilibrium (mLD) was calculated using LIAN version 3.7, applying a Monte Carlo test with 100,000 re-sampling steps (Haubold \& Hudson, 2000). In the LIAN analysis only samples with complete haplotypes were included. For both species the dominant and single haplotypes were compared within catchments to identify any significant linkage disequilibrium (mLD) using LIAN (Haubold \& Hudson, 2000) and $F_{S T}$ using FSTAT version 2.9.3.2 (Goudet, 1995).

Bottleneck (Piry, Luikart, \& Cornuet) was used to test for an excess of heterozygosity brought about by the loss of rare alleles following a population bottleneck. A two-phase mutational (TPM) model of $70 \%$ stepwise and $30 \%$ non-stepwise mutations and run 1000 iterations was used. In addition, the allele frequency distribution for all loci was examined for a 'mode shift' in the distribution (Luikart, Allendorf, Cornuet, \& Sherwin, 1998). To investigate parasite population genetic structure, the Bayesian clustering software, STRUCTURE version 2.3.4

309 (Pritchard, Stephens, \& Donnelly, 2000) was used to investigate whether haplotypes for each 310 species clustered according to geographical origin and/or within time periods. The analysis 311 was run 20 times for $\mathrm{K}=1$ to 15 for 100,000 Monte Carlo Markov Chain (MCMC) iterations 312 after a burn-in period of 10,000 using the admixture model and correlated allele frequencies. 313 The second order rate of change of $\mathrm{LnP}[\mathrm{D}], \Delta \mathrm{K}$ was calculated according to the method of 
314 Evanno et al. (Evanno, Regnaut, \& Goudet, 2005) to determine the most likely K (most likely 315 number of populations). CLUMPP version 1.1.2 (Jakobsson \& Rosenberg, 2007) was used to 316 facilitate the interpretation of population genetic results using files generated with 317 STRUCTURE HARVESTER Web v0.6.94 (Earl \& vonHoldt, 2011) and Distruct 1.1 318 (Rosenberg, 2004) was used to visualize the structure plots with the data generated with 319 CLUMPP. Statistical analysis of molecular epidemiological and population genetic 320 parameters was done using non-parametric methods as indicated in the results-section using 321 STATA v12.1 (StataCorp, USA). QGIS 2.18.24 (OpenSource Geospatial Foundation) was

322 used to map the villages and households and maps were constructed with spatial layers from 323 DIVA-GIS ("Diva GIS country level data,").

\section{RESULTS}

\section{Multiplicity of Infection}

Multiplicity of infection (MOI), determined by genotyping highly polymorphic markers and counting the numbers of alleles in each infection, is a proxy measure of the intensity of transmission. $\mathrm{MOI}$ in all areas was lower in all areas post-LLIN distribution decreasing from 1.8 to $1.3(p=0.0463$ Mann Whitney $U$ test $)$ and 2.0 to $1.4(p=0.0495$ Mann Whitney $U$ test $)$, respectively for $P$. falciparum and $P$. vivax. This corresponds with a lower proportion of multiclonal infections, except for $P$. falciparum in ESP in 2012-13 (Figure 1 and Table S1). Despite an increase in PCR prevalence of $P$. vivax in Madang Province in 2014, few multiclonal infections were detected (Figure 1 and Table S1).

\section{Microsatellite haplotypes}

We then genotyped parasite isolates at microsatellite loci to generate multilocus haplotypes for population genetic analyses. Multilocus haplotypes with at least five loci successfully genotyped (out of 9 for $P$. falciparum and 10 for $P$. vivax) were constructed for $860 P$. falciparum samples (300 previously published) and 755 P. vivax samples (202 previously published) (Jennison et al., 2015; Schultz et al., 2010) (Table S1). Despite having genotyped the samples that were identified as $\mathrm{MOI}=1$ by $p f m s p 2$, pvmsp1f3 and pvMS2/MS16 genotyping, $31 \%$ of $P$. falciparum samples and $49 \%$ of $P$. vivax samples had more than one allele for at least one microsatellite locus, suggesting multiple clone infection and the increased resolution of the microsatellite panel. From these we created dominant haplotypes (Schultz et al., 2010). No significant changes in multilocus Linkage Disequilibrium (mLD) were found when comparing single vs. all haplotypes combined within each study (Table S2). Low genetic differentiation was found between single and dominant haplotypes for $P$. falciparum in MAD2014 $\left(F_{\mathrm{ST}}=0.063, \mathrm{p}=0.58\right)$, however, this can be explained by small 
sample size ( $\mathrm{n}=9$ dominant haplotypes). For $P$. vivax, low differentiation between single and dominant haplotypes in ESP $2012\left(F_{\mathrm{ST}}=0.041, \mathrm{p}=0.33\right)$, was explained by a cluster of closely related haplotypes, all reconstructed from dominant alleles), which are described in more detail below. The fact that these related haplotypes were independently constructed from dominant haplotypes provides additional confidence in the allele-calling strategy. All other comparisons (within each province for at each time point) showed negligible genetic differentiation between single and dominant haplotypes. Therefore, the haplotypes were combined for further analysis.

\section{Reduction in $P$. falciparum but not $P$. vivax genetic diversity post-LLIN}

Based on the microsatellite haplotypes $(n=860)$, the genetic diversity of $P$. falciparum populations was modestly but significantly lower post-LLIN compared to the earlier time points for pre-LLIN populations (ESP1 and 22005 and MAD 2006). Mean heterozygosity for $P$. falciparum over all areas combined decreased significantly from $0.76 \pm 0.1$ to $0.71 \pm 0.1$ (Mann-Whitney $U$ test $p=0.036$; Table S1) and allelic richness from $7.7 \pm 2.2$ to $6.5 \pm 2.1$ (Mann-Whitney $U$ test $p=0.014$; Figure 2). These parameters also showed a small but insignificant decline for the provinces analysed individually (Figure 2, $p>0.05$ ). For $P$. vivax, overall genetic diversity remained high (post-LLIN $R s=12.5 ; H e=0.85$, Table $S 1$ ), but slightly different results were seen in each province (Figure 2, Table S1). In Madang Province after the distribution of LLINs, He values slightly increased from $0.85 \pm 0.07$ in 2006 to $0.88 \pm 0.04$ in $2014(p=0.3$, Table S1), with high allelic richness (pre-LLIN Rs $12.2 \pm 4.0$ vs post-LLIN $14.0 \pm 3.4 ; p=0.2$ ). Whereas in East Sepik Province, $P$. vivax genetic diversity decreased but not significantly with $\mathrm{He}$ values of $0.83 \pm 0.09$ to $\mathrm{He} 0.80 \pm 0.08$ (2-sample t-test, $\mathrm{p}=0.48$ ) and $R s$ values of $11.1 \pm 3.5$ vs post-LLIN Rs $9.8 \pm 3.5$ (2-sample t-test, $p=0.33$ ) (Table $S 1$, Figure 2). No significant correlation was found between prevalence (by PCR) and heterozygosity, allelic richness, mean $\mathrm{MOI}$, or proportion of multiple clone infections, for either species (data not shown).

\section{Significant multilocus linkage disequilibrium (mLD) for both $P$. falciparum and $P$. vivax post-LLIN}

For $P$. falciparum, matching haplotypes (allowing missing loci) were seen in all post-LLIN datasets and the pre-LLIN ESP2 2005 dataset. However, for P. vivax, matching haplotypes (allowing missing loci) were rarely seen and only in post-LLIN data sets. Among the 332 complete $P$. falciparum multilocus haplotypes (9-loci successfully genotyped) from all study sites, 16 repeated haplotypes were found, with 11 haplotypes represented two times, three represented three times, and two represented four times. Clonal haplotypes were always found within the same year and province, and in all cases except one in the same catchment 
area, but not always in the same village (7/16 haplotypes found in neighbouring villages). In ESP2 2005, one clonal haplotype was found in two villages (Yenigo and Sengo, Figure 1) from different catchment areas, roughly $40 \mathrm{~km}$ apart. Among the 303 complete P. vivax multilocus haplotypes (10-loci), two haplotypes were repeated, with one haplotype represented two times (in two different villages in ESP 2012), and one represented four times (three in one village, one in a neighbouring village in MAD 2010).

To investigate whether inbreeding was present in these populations (Smith, Smith, O'Rourke, \& Spratt, 1993), non-random associations among the microsatellite loci (mLD) were calculated for all complete and unique haplotypes. Whilst mLD was absent from most preLLIN populations, significant $\mathrm{mLD}$ was observed in $P$. falciparum and $P$. vivax infections post-LLIN (Table 1). In ESP 2012-13, mLD was high with unique infections indicating the circulation of closely related haplotypes in the population, suggesting near clonal transmission (low recombination between diverse clones and high levels of inbreeding) or the presence of high proportions of meiotic siblings among isolates (Bright et al., 2014; Smith et al., 1993), as observed in the village of Sunuhu (Table S3). Low, but significant mLD was found for $P$. falciparum in Madang in 2006 (Table 1), however this population was structured (Schultz et al., 2010), resulting in a phenomenon called the Wahlund effect, confirmed by the fact that linkage equilibrium was restored when $\mathrm{mLD}$ was analysed separately for subpopulations (Jennison et al., 2015; Wahlund, 1928) (Table S4). In post-LLIN Madang, the observed $\mathrm{mLD}$ for $P$. falciparum is not the result of subpopulation structure, as significant $m L D$ remained in the subpopulations (Table S4). In the Mugil area in 2014, significant mLD for $P$. falciparum remains due to the circulation of a few very closely related haplotypes in the Megiar village (Table S4, Dataset 1).

\section{Population Bottleneck for $P$. falciparum but not $P$. vivax post-LLIN}

Bottleneck analysis was performed using a two-phase mutational model (TPM) and testing for heterozygosity excess with a 2-tailed Wilcoxon sign rank test (see Materials and Methods). Significant heterozygosity excess was observed for $P$. falciparum in ESPII 2005 (but not in ESPI 2005), MAD 2006 and 2010 populations $(p=0.020, p=0.049$ and $p=0.010$, respectively). However, for $P$. vivax significant heterozygosity excess was only observed in a single pre-LLIN population, Wosera 2005 ( $p=0.042$, these samples were collected in the relatively dry season) and not in any of the other $P$. vivax populations. Despite finding significant heterozygosity excess, a mode-shifted distribution of allele frequencies (as is frequently observed in bottlenecked populations) was not observed in any of the time points and provinces. 
417 Contrasting and dynamic patterns of population structure for both $\boldsymbol{P}$. falciparum and 418 P. vivax

419 As previously described, for $P$. falciparum, low to moderate genetic differentiation was seen 420 between the Wosera 2005 (ESP1) and Madang 2006 studies (Jennison et al., 2015; Schultz 421 et al., 2010) (Figure 3, S1). Comparisons were not done between Pf 2005/6 and other Pf 422 populations as it was not possible to calibrate data through combining allele calls before 423 binning (see Materials and Methods). Post-LLIN, there remains low to moderate genetic 424 differentiation between ESP (2012-2013) and Madang (2014) (Figure 3, S1). However, there 425 was little genetic differentiation of East Sepik $P$. falciparum populations pre-LLIN (ESP2 426 2005) compared to post-LLIN (2012-13), nor between Madang 2010 and 2014 populations 427 (Figure 3, S1). For P. vivax, a different pattern can be seen, with low genetic differentiation 428 between provinces pre-LLIN, which increases in the post LLIN-studies (Figure 3, S1). Similar 429 to $P$. falciparum, within province genetic differentiation between the different time points does 430 not increase post-LLIN.

431 Population genetic structure was further investigated by Bayesian cluster analysis using 432 STRUCTURE (Pritchard et al., 2000). Haplotypes or populations with ancestry in more than 433 one cluster are considered admixed and indicates that substantial gene flow occurs between defined geographic areas. Our analysis identified three $P$. falciparum and three $P$. vivax clusters (Figure 4, S2). The clustering patterns show that the $P$. falciparum populations in later years are more mixed than the populations of 2005/2006, where populations clustered according to geographical locations including amongst the three catchment areas within Madang Province (Figure 4, S2) (Schultz et al., 2010). On the contrary, P. vivax populations were very diverse and displayed little population structure in all time points, despite the increase in differentiation between ESP and MAD post-LLIN populations (Figures 4 and 5).

441 For $P$. vivax, as we observed significant mLD post-LLIN in ESP, local phylogenetic analysis 442 was conducted. This supports focal transmission as shown by the clustering of haplotypes 443 from the same village: Sunuhu (Figure 5). The STRUCTURE analysis also shows some 444 evidence of this inbred cluster (Figure 4B). Interestingly, this village had the highest 445 prevalence in the region in the $2012-13$ survey (36\% infected with $P$. vivax by qPCR 446 compared to $0.5-9 \%$ in other villages (Kattenberg et al., 2020). In Sunuhu, clonal and closely 447 related haplotypes ( $\leq 2$ unmatched alleles) were observed in $48 \%(11 / 23)$ of the haplotypes 448 from that village (see supplementary file 1). The 11 closely related haplotypes were observed 449 throughout the village, were not clustered in neighbouring households, and were not 450 associated with participant characteristics ( $p>0.05$ ), such as age and sex (Table S3). 
452 Parasite genetic diversity has been proposed as a key indicator of malaria transmission 453 dynamics to track control and elimination progress (Dalmat et al., 2019). This demands an 454 evaluation of whether genotyping provides insights about changes in transmission following intensified malaria control efforts. Here we have monitored the population genetics of $P$. falciparum and $P$, vivax over an eight-year period of LLIN-induced transmission decline in PNG, an area of high year round transmission. Despite large reductions in parasite prevalence and multiple infections from very high to low/moderate levels (Kattenberg et al., 2020; Koepfli et al., 2017; Koepfli et al., 2015), we show that population genetic changes were minimal with populations remaining diverse and unstructured. $P$. falciparum diversity decreased somewhat, though remained high relative to other malaria endemic areas outside Africa (Anderson et al., 2000; Branch et al., 2011; Chenet et al., 2012; Chenet et al., 2015; dalla Martha, Tada, Ferreira, da Silva, \& Wunderlich, 2007; Noviyanti et al., 2015; OrjuelaSanchez et al., 2009; Orjuela-Sanchez et al., 2013; Pava et al., 2017; Susomboon et al., 2008), whereas $P$. vivax diversity was unchanged throughout the study period. Surprisingly, $P$. falciparum populations that were structured pre-LLIN $(2005,2006)$ (Jennison et al., 2015; Schultz et al., 2010), were unstructured post-LLIN (2010, 2014), although a reduction in multiple infections and an increase in multilocus LD due to clonal haplotypes were detected. For $P$. vivax, there was also no evidence of population structure after LLIN, however increasing pairwise genetic differentiation within and between provinces was observed and clonal transmission and inbreeding had emerged in at least one village. These results demonstrate that declining transmission does not result in an immediate decrease in overall population diversity nor an increase in population structure. Sustained low transmission may be needed to observe these changes using these small panels of microsatellite markers. However, changes in multiple infection prevalence and multilocus LD indicate increasing heterogeneity in transmission within populations. These results have implications with respect to monitoring changing transmission for any pathogen using population genetic approaches.

Nkhoma and colleagues previously reported a limited impact on $P$. falciparum diversity following a decrease from moderate to low transmission on the Thai-Myanmar border over 10 years. However, as we have observed in PNG, there was an increase in multilocus LD which and decreasing proportions of multiclonal infections (Nkhoma et al., 2013). Daniels et al. have also reported decreasing multiclonal infections, increasing proportions of repeated genotypes and multilocus LD, and long-term persistence of particular clones in Senegal (Daniels et al., 2013). These studies utilised 96 and 24 biallelic SNP markers respectively, 
compared to our study using a small number of microsatellite markers. Similar panels of SNPs may reveal additional insights in the PNG context. As for P. vivax, Gunawardena and colleagues also reported sustained high $P$. vivax microsatellite diversity and multiclonal infections during a five-year period as the country progressed towards malaria elimination (Gunawardena et al., 2014). Population genetic signals of declining transmission might take longer to emerge for $P$. vivax due to new blood-stage infections from the hypnozoite reservoir and could explain why we only observed mLD in one village of East Sepik.

In PNG, the limited decline in P. falciparum diversity and loss of parasite population structure after LLIN distribution indicates that there may be increased gene flow between the sampled parasite populations, which was unexpected. Population structure prior to intensified control was thought to be the result of limited historical human migration due to the rugged terrain and lack of direct transport connections (Mueller, Bockarie, Alpers, \& Smith, 2003; Riley, 1983) or population bottlenecks due to past control efforts or emergence of drug resistance (Anderson et al., 2000; Jennison et al., 2015; Schultz et al., 2010; Tessema et al., 2015). Changes

in first-line treatmen policies, for example the introduction of sulphadoxine/pyrimethamine (SP) in the early 2000's and artemether-lumefantrine in 2008, might have played a role in shaping parasite population structure ( $\mathrm{Mu}$ et al., 2005). Chloroquine $(\mathrm{CQ})$ and/or SP resistance (near fixation of resistant pfmdr1 and pfdhps resistant alleles were observed in the same areas (Barnadas et al., 2015; Koleala et al., 2015; Mita et al., 2018)) and may have contributed to the observed bottleneck and mLD in pre-LLIN $P$. falciparum populations, with consequent reductions in effective population size, while outcrossing due to high transmission preserved within-population genetic diversity as the resistance mutation spread (Mita et al., 2018). From 2000 to 2011 the PNG population increased by over two million people (National Census Report, 2011), and local observations suggest that large numbers of migrants from East Sepik have moved into Madang in the last decade seeking employment opportunities. As a result, post-LLIN, greater connectivity between human populations may have broken down $P$. falciparum population structure and maintained high gene flow between $P$. vivax populations (Fola et al., 2018).

For the post-LLIN East Sepik P. vivax population where prevalence dropped to below $5 \%$, significant $m L D$ was observed resulting from very closely related parasite strains circulating in a residual pocket of relatively high transmission within a single village. This suggests considerable inbreeding of parasites in that village, in a background of high genetic diversity resulting in a lack of evidence of a bottleneck at the population level. This paradoxical signature of significant $\mathrm{mLD}$ with high diversity and a considerable proportion of multiple clone infections of $P$. vivax appears to be a hallmark of lower transmission areas (Barry et al., 2015; Batista et al., 2015; Chenet et al., 2012; Delgado-Ratto et al., 2016; Delgado-Ratto 
et al., 2014; Ferreira et al., 2007; Hong et al., 2016; Noviyanti et al., 2015; Orjuela-Sanchez et al., 2013). Similar to $P$. falciparum populations though, there was a correlation between mLD and prevalence of infection for $P$. vivax. This shows that reductions in multiclonal infections and $\mathrm{mLD}$ is an earlier indicator of reduced transmission than genetic diversity and population structure (for both species).

Multilocus LD in post-LLIN $P$. vivax populations was explained by both focal clonal transmission and inbreeding, as similarly observed in other studies in Peru, Vietnam, and Papua Indonesia (Delgado-Ratto et al., 2014; Hong et al., 2016; Noviyanti et al., 2015). The explanation for this observation will most likely lie in unique $P$. vivax characteristics related to hypnozoites, relapses and transmission dynamics (Abdullah et al., 2013; Bright et al., 2014; Chen, Auliff, Rieckmann, Gatton, \& Cheng, 2007; Delgado-Ratto et al., 2014; Ferreira et al., 2007; Fola et al., 2018; Iwagami et al., 2012; White, 2011). At high transmission (e.g. preLLIN) with high prevalence and high multiplicity of infection, these clusters of similar haplotypes might also occur, but could be obscured due to sampling limitations and the large number of different haplogroups circulating at the same time. As transmission declines, infections have fewer clones and the diversity of the hypnozoite reservoir decreases, resulting in fewer circulating haplogroups, lower levels of recombination between distinct genomes and more frequent clonal transmission and inbreeding, resulting in increased $\mathrm{mLD}$ as in this and other studies (Barry et al., 2015; Batista et al., 2015; Chenet et al., 2012; Delgado-Ratto et al., 2016; Delgado-Ratto et al., 2014; Ferreira et al., 2007; Hong et al., 2016; Noviyanti et al., 2015; Orjuela-Sanchez et al., 2013). These signatures are more likely to be seen in a population with more sustained low transmission such as was the case for the East Sepik Province. In this region, malaria transmission is heterogeneous between villages. Besides the national malaria control program, other initiatives were also distributing LLINs in East Sepik Province prior to the nationwide distribution (Hetzel et al., 2014; Hetzel et al., 2012, Hetzel et al., 2016) suggesting that longer term sustained control efforts have been effective.

550 Considerable variance in the impact of the LLIN program was observed in the two provinces. 551 In Madang, whilst $P$. falciparum rates steadily declined over the entire study period, there 552 was a resurgence of submicroscopic P. vivax infections in 2014 (Koepfli et al., 2017). 553 Although multiplicity of infection remained low, the lack of $\mathrm{mLD}$ and population structure 554 suggests that this is not due to an outbreak, but more likely the residual $P$. vivax population was able to gain a foothold once again despite the intensive control efforts. In addition, an increase in the prevalence of pvdhfr triple and quadruple mutants, related with SP resistance, were observed in Madang province in 2010 (Barnadas et al., 2015), and a high proportion of 558 resistant parasites could be a possible explanation for the higher infection prevalence by 
2014. Different studies have shown that selective pressure of drugs such as CQ and/or SP 560 have had an impact on shaping worldwide $P$. vivax populations in recent history (Hupalo et 561 al., 2016; Pearson et al., 2016). However, a population bottleneck as seen in P. falciparum 562 populations (Mita et al., 2018) did not occur in P. vivax populations of PNG. Malaria control 563 had a greater impact on $P$. vivax prevalence in East Sepik and population structure was 564 observed in one village post-LLIN.

565 This study has some limitations related to sampling and the genotyping approach used. The samples were collected in serial cross-sectional surveys over a period of malaria control initiated at different times in the two provinces. Fewer years of sustained control pressure compared to East Sepik might explain why, despite substantial prevalence decline in Madang Province by 2010, we did not observe any signature of changing population structure. Whilst, in East Sepik 2012, a cluster of closely related parasites was observed in one village suggesting more focal transmission than previous years. The microsatellite panels were selected as these have been the gold standard genotyping tool for large-scale malaria population genetic studies for many years (Anderson et al., 1999; Imwong et al., 2006; Karunaweera et al., 2006). However, they are limited in number (less than one per chromosome), rapidly evolving and prone to error. Although these markers are extremely useful for measuring parasite population structure on local and global scales (Auburn \& Barry, 2017; Barry et al., 2015; Koepfli \& Mueller, 2017; Sutton, 2013), they are not ideal for cross-study comparisons due to the difficultly in calibrating alleles from different data sources. The lack of raw data from the previously published $P$. falciparum study (Schultz et al., 2010), prevented the direct comparison of haplotypes and thus the analysis of population structure between the earlier $P$. falciparum time points for the East Sepik II (Wosera) and

582 Madang populations. Furthermore, dominant haplotypes derived from multiple clone 583 infections can be reconstructed incorrectly, thus inflating diversity values (Messerli, Hofmann, 584 Beck, \& Felger, 2017). However, only haplotype-based analyses such as multilocus LD and phylogenetic analysis were vulnerable to these possible artefacts, and would result in an overestimate of diversity and underestimate of LD. All other analyses were conducted using mean values across markers or allele frequencies and thus limit the impact of such errors. Moreover, the dataset included a large proportion of single infection haplotypes in all populations, and the detected clones included dominant haplotypes suggesting that allele 590 calling was reliable. Finally, the highly polymorphic and rapidly evolving nature of 591 microsatellite markers (Ellegren, 2004) may limit the resolution of the population genetic 592 parameters such as population level diversity and population structure in high transmission 593 areas (Branch et al., 2011). This may both lead to false assignment of unrelated parasites 594 (e.g. from different provinces) as related and reduce the detection of truly related parasites 
595 (identical by descent), both of which would result in a lack of population structure. Other 596 more stable markers, such as SNPs (Baniecki et al., 2015; R. Daniels et al., 2008) or whole 597 genome sequencing (Hupalo et al., 2016; Miotto et al., 2013; Mu et al., 2005; Pearson et al., 598 2016; Volkman et al., 2007) may be more sensitive and specific to detect changes in parasite 599 population structure.

600 According to the data presented, in two high transmission provinces of PNG, recent 601 reductions in malaria transmission result in limited changes in parasite population diversity 602 and structure as determined by microsatellite markers. The high parasite diversity and lack of 603 population structure are consistent with both species maintaining a large and evolutionarily fit 604 population immediately after prevalence decline suggesting the gains made are fragile. 605 However, fewer multiclonal infections, and the emergence of significant $\mathrm{mLD}$ for both species 606 indicates there is focally interrupted transmission and suggests that these parameters may 607 be useful markers for measuring control impact and early changes in parasite population 608 structure with intervention. The results were in contrast to our expectations of decreasing 609 diversity and increasing population structure (Jennison et al., 2015) and show that long term 610 sustained control efforts may need to be maintained to observe significant change in 611 population structure, at least as measured by the microsatellite panels used in this study. 612 The PNG national malaria control program has made an impact on the malaria burden 613 through the substantial reductions in infections circulating in the community (Hetzel et al., 614 2016; Kattenberg et al., 2020; Koepfli et al., 2017), however it appears that this has not been 615 sustained long enough for the underlying parasite population to fragment. Finally, this study 616 demonstrates how parasite population genetics can inform whether malaria intervention has 617 reduced and fragmented the parasite population or if significantly more control effort will be 618 required to do so.

\section{ACKNOWLEDGEMENTS}

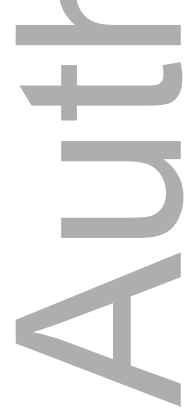

This article is protected by copyright. All rights reserved 
620 We are grateful to the people who participated in the study, and for the support and 621 collaboration of the participating communities. We appreciate the efforts of the laboratory, 622 field team members and support staff from the Papua New Guinea Institute of Medical 623 Research for their involvement in the included studies. This research was supported by 624 National Health and Medical Research Council (NHMRC) of Australia Project Grants 625 (1010069 and 1027108), the International Centres of Excellence in Malaria Research 626 (ICEMR) for the South West Pacific, NIH U19 Al089686 and a Bill \& Melinda Gates 627 Foundation Grant (TransEpi Consortium). IM and LR are supported by NHMRC Research 628 Fellowships.

\section{DATA AVAILABILITY STATEMENT}

631 The data that support the findings of this study are openly available in Dryad at https://doi.org/10.5061/dryad.37pvmcvfh

\section{REFERENCES}

Abdullah, N. R., Barber, B. E., William, T., Norahmad, N. A., Satsu, U. R., Muniandy, P. K., . . Auburn, S. (2013). Plasmodium vivax population structure and transmission dynamics in Sabah Malaysia.PLoS One, 8(12), e82553. doi:10.1371/journal.pone.0082553

Anderson, T. J., Haubold, B., Williams, J. T., Estrada-Franco, J. G., Richardson, L., Mollinedo, R., .. Day, K.P. (2000). Microsatellite markers reveal a spectrum of population structures in the malaria parasite Plasmodium falciparum. Mol Biol Evol, 17(10), 1467-1482. doi:10.1093/oxfordjournals.molbev.a026247

Anderson, T. J., Su, X. Z., Bockarie, M., Lagog, M., \& Day, K. P. (1999). Twelve microsatellite markers for characterization of Plasmodium falciparum from finger-prick blood samples. Parasitology, 119 (Pt 2), 113-125. doi:10.1017/s0031182099004552

Argimon, S., Abudahab, K., Goater, R. J. E., Fedosejev, A., Bhai, J., Glasner, C., . . Aanensen, D. M. (2016). Microreact: visualizing and sharing data for genomic epidemiology and phylogeography. Microb Genom, 2(11), e000093. doi:10.1099/mgen.0.000093

Arnott, A., Barnadas, C., Senn, N., Siba, P., Mueller, I., Reeder, J. C., \& Barry, A. E. (2013). High genetic diversity of Plasmodium vivax on the north coast of Papua New Guinea. Am J Trop Med Hyg, 89(1), 188-194. doi:10.4269/ajtmh.12-0774

Arnott, A., Barry, A. E., \& Reeder, J. C. (2012). Understanding the population genetics of Plasmodium vivax is essential for malaria control and elimination. Malar J, 11, 14. doi:10.1186/1475-2875$11-14$

This article is protected by copyright. All rights reserved 
Auburn, S., \& Barry, A. E. (2017). Dissecting malaria biology and epidemiology using population genetics and genomics. Int J Parasitol, 47(2-3), 77-85. doi:10.1016/j.ijpara.2016.08.006 Babiker, H. A., Ranford-Cartwright, L. C., Currie, D., Charlwood, J. D., Billingsley, P., Teuscher, T., \& Walliker, D. (1994). Random mating in a natural population of the malaria parasite Plasmodium falciparum. Parasitology, 109 ( Pt 4), 413-421. doi:10.1017/s0031182000080665 Baniecki, M. L., Faust, A. L., Schaffner, S. F., Park, D. J., Galinsky, K., Daniels, R. F., . . Sabeti, P. C. (2015). Development of a single nucleotide polymorphism barcode to genotype Plasmodium vivax infections. PLoS Neg/ Trop Dis, 9(3), e0003539. doi:10.1371/journal.pntd.0003539

Barnadas, C., Timinao, L., Javati, S., Iga, J., Malau, E., Koepfli, C., . . Mueller, I. (2015). Significant geographical differences in prevalence of mutations associated with Plasmodium falciparum and Plasmodium vivax drug resistance in two regions from Papua New Guinea. Malar J, 14, 399. doi:10.1186/s12936-015-0879-9

Barry, A. E., Schultz, L., Senn, N., Nale, J., Kiniboro, B., Siba, P. M., . . Reeder, J. C. (2013). High levels of genetic diversity of Plasmodium falciparum populations in Papua New Guinea despite variable infection prevalence. Am J Trop Med Hyg, 88(4), 718-725. doi:10.4269/ajtmh.120056

Barry, A. E., Waltmann, A., Koepfli, C., Barnadas, C., \& Mueller, I. (2015). Uncovering the transmission dynamics of Plasmodium vivax using population genetics. Pathog Glob Health, 109(3), 142152. doi:10.1179/2047773215Y.0000000012

Batista, C. L., Barbosa, S., Da Silva Bastos, M., Viana, S. A., \& Ferreira, M. U. (2015). Genetic diversity of Plasmodium vivax over time and space: a community-based study in rural Amazonia. Parasitology, 142(2), 374-384. doi:10.1017/S0031182014001176

Bei, A. K., Niang, M., Deme, A. B., Daniels, R. F., Sarr, F. D., Sokhna, C., .. Toure-Balde, A. (2018). Dramatic Changes in Malaria Population Genetic Complexity in Dielmo and Ndiop, Senegal, Revealed Using Genomic Surveillance. J Infect Dis, 217(4), 622-627. doi:10.1093/infdis/jix580

Betuela, I., Maraga, S., Hetzel, M. W., Tandrapah, T., Sie, A., Yala, S., ... Mueller, I. (2012). Epidemiology of malaria in the Papua New Guinean highlands. Trop Med Int Health, 17(10), 1181-1191. doi:10.1111/j.1365-3156.2012.03062.x

Branch, O. H., Sutton, P. L., Barnes, C., Castro, J. C., Hussin, J., Awadalla, P., \& Hijar, G. (2011). Plasmodium falciparum genetic diversity maintained and amplified over 5 years of a low transmission endemic in the Peruvian Amazon. Mol Biol Evol, 28(7), 1973-1986. doi:10.1093/molbev/msq311

Bright, A. T., Manary, M. J., Tewhey, R., Arango, E. M., Wang, T., Schork, N. J., . . Winzeler, E. A. (2014). A high resolution case study of a patient with recurrent Plasmodium vivax infections 
shows that relapses were caused by meiotic siblings. PLoS Negl Trop Dis, 8(6), e2882. doi:10.1371/journal.pntd.0002882

Chen, N., Auliff, A., Rieckmann, K., Gatton, M., \& Cheng, Q. (2007). Relapses of Plasmodium vivax infection result from clonal hypnozoites activated at predetermined intervals. J Infect Dis, 195(7), 934-941. doi:10.1086/512242

Chenet, S. M., Schneider, K. A., Villegas, L., \& Escalante, A. A. (2012). Local population structure of Plasmodium: impact on malaria control and elimination. Malar J, 11, 412. doi:10.1186/14752875-11-412

Chenet, S. M., Taylor, J. E., Blair, S., Zuluaga, L., \& Escalante, A. A. (2015). Longitudinal analysis of Plasmodium falciparum genetic variation in Turbo, Colombia: implications for malaria control and elimination. Malar J, 14, 363. doi:10.1186/s12936-015-0887-9

Cornejo, O. E., \& Escalante, A. A. (2006). The origin and age of Plasmodium vivax. Trends Parasitol, 22(12), 558-563. doi:10.1016/j.pt.2006.09.007

dalla Martha, R. C., Tada, M. S., Ferreira, R. G., da Silva, L. H., \& Wunderlich, G. (2007). Microsatellite characterization of Plasmodium falciparum from symptomatic and non-symptomatic infections from the Western Amazon reveals the existence of non-symptomatic infectionassociated genotypes. Mem Inst Oswaldo Cruz, 102(3), 293-298. doi:10.1590/s007402762007005000044

Dalmat, R., Naughton, B., Kwan-Gett, T. S., Slyker, J., \& Stuckey, E. M. (2019). Use cases for genetic epidemiology in malaria elimination. Malar J, 18(1), 163. doi:10.1186/s12936-019-2784-0

Daniels, R., Volkman, S. K., Milner, D. A., Mahesh, N., Neafsey, D. E., Park, D. J., . . Wiegand, R. C. (2008). A general SNP-based molecular barcode for Plasmodium falciparum identification and tracking. Malar J, 7, 223. doi:10.1186/1475-2875-7-223

Daniels, R. F., Schaffner, S. F., Wenger, E. A., Proctor, J. L., Chang, H. H., Wong, W., . . Wirth, D. F. (2015). Modeling malaria genomics reveals transmission decline and rebound in Senegal. Proc Natl Acad Sci U S A, 112(22), 7067-7072. doi:10.1073/pnas.1505691112

Delgado-Ratto, C., Gamboa, D., Soto-Calle, V. E., Van den Eede, P., Torres, E., Sanchez-Martinez, L., . . . D'Alessandro, U. (2016). Population Genetics of Plasmodium vivax in the Peruvian Amazon. PLoS Negl Trop Dis, 10(1), e0004376. doi:10.1371/journal.pntd.0004376

Delgado-Ratto, C., Soto-Calle, V. E., Van den Eede, P., Gamboa, D., Rosas, A., Abatih, E. N., ... D'Alessandro, U. (2014). Population structure and spatio-temporal transmission dynamics of Plasmodium vivax after radical cure treatment in a rural village of the Peruvian Amazon. Malar J, 13, 8. doi:10.1186/1475-2875-13-8

Diva GIS country level data. Retrieved from http://www.diva-gis.org/gdata 
Earl, D. A., \& vonHoldt, B. M. (2011). STRUCTURE HARVESTER: a website and program for visualizing STRUCTURE output and implementing the Evanno method. Conservation Genetics Resources, 4(2), 359-361. doi:10.1007/s12686-011-9548-7

Ellegren, H. (2004). Microsatellites: simple sequences with complex evolution. Nat Rev Genet, 5(6), 435-445. doi:10.1038/nrg1348

Evanno, G., Regnaut, S., \& Goudet, J. (2005). Detecting the number of clusters of individuals using the software STRUCTURE: a simulation study. Mol Ecol, 14(8), 2611-2620. doi:10.1111/j.1365294X.2005.02553.x

Feachem, R. G., Phillips, A. A., Hwang, J., Cotter, C., Wielgosz, B., Greenwood, B. M., . . Snow, R. W. (2010). Shrinking the malaria map: progress and prospects. Lancet, 376(9752), 1566-1578. doi:10.1016/S0140-6736(10)61270-6

Ferreira, M. U., Karunaweera, N. D., da Silva-Nunes, M., da Silva, N. S., Wirth, D. F., \& Hartl, D. L. (2007). Population structure and transmission dynamics of Plasmodium vivax in rural Amazonia. J Infect Dis, 195(8), 1218-1226. doi:10.1086/512685

Fola, A. A., Harrison, G. L. A., Hazairin, M. H., Barnadas, C., Hetzel, M. W., Iga, J., . . Barry, A. E. (2017). Higher Complexity of Infection and Genetic Diversity of Plasmodium vivax Than Plasmodium falciparum Across All Malaria Transmission Zones of Papua New Guinea. Am J Trop Med Hyg, 96(3), 630-641. doi:10.4269/ajtmh.16-0716

Fola, A. A., Nate, E., Abby Harrison, G. L., Barnadas, C., Hetzel, M. W., Iga, J., . . Barry, A. E. (2018). Nationwide genetic surveillance of Plasmodium vivax in Papua New Guinea reveals heterogeneous transmission dynamics and routes of migration amongst subdivided populations. Infect Genet Evol, 58, 83-95. doi:10.1016/j.meegid.2017.11.028

Gatei, W., Kariuki, S., Hawley, W., ter Kuile, F., Terlouw, D., Phillips-Howard, P., . . Shi, Y. P. (2010). Effects of transmission reduction by insecticide-treated bed nets (ITNS) on parasite genetics population structure: I. The genetic diversity of Plasmodium falciparum parasites by microsatellite markers in western Kenya. Malar J, 9, 353. doi:10.1186/1475-2875-9-353

Genton, B., al-Yaman, F., Beck, H. P., Hii, J., Mellor, S., Narara, A., ... Alpers, M. P. (1995). The epidemiology of malaria in the Wosera area, East Sepik Province, Papua New Guinea, in preparation for vaccine trials. I. Malariometric indices and immunity. Ann Trop Med Parasitol, 89(4), 359-376. doi:10.1080/00034983.1995.11812965

Genton, B., Hii, J., al-Yaman, F., Paru, R., Beck, H. P., Ginny, M., . . Alpers, M. P. (1994). The use of untreated bednets and malaria infection, morbidity and immunity. Ann Trop Med Parasitol, 88(3), 263-270. doi:10.1080/00034983.1994.11812866

This article is protected by copyright. All rights reserved 
Gilabert, A., Otto, T. D., Rutledge, G. G., Franzon, B., Ollomo, B., Arnathau, C., ... Rougeron, V. (2018). Plasmodium vivax-like genome sequences shed new insights into Plasmodium vivax biology and evolution. PLoS Biol, 16(8), e2006035. doi:10.1371/journal.pbio.2006035

Glaubitz, J. C. convert: A user-friendly program to reformat diploid genotypic data for commonly used population genetic software packages.

Goudet, J. (1995). FSTAT (Version 1.2): A Computer Program to Calculate F-Statistics. Journal of Heredity, 86(6), 485-486.

Grad, Y. H., \& Lipsitch, M. (2014). Epidemiologic data and pathogen genome sequences: a powerful synergy for public health. Genome Biology, 15(11), 538. doi:10.1186/s13059-014-0538-4

Gunawardena, S., Ferreira, M. U., Kapilananda, G. M., Wirth, D. F., \& Karunaweera, N. D. (2014). The Sri Lankan paradox: high genetic diversity in Plasmodium vivax populations despite decreasing levels of malaria transmission. Parasitology, 141(7), 880-890. doi:10.1017/S0031182013002278

Haubold, B., \& Hudson, R. R. (2000). LIAN 3.0: detecting linkage disequilibrium in multilocus data. Linkage Analysis. Bioinformatics, 16(9), 847-848. doi:10.1093/bioinformatics/16.9.847

Hedtke, S. M., Kuesel, A. C., Crawford, K. E., Graves, P. M., Boussinesq, M., Lau, C. L., . . Grant, W. N. (2019). Genomic Epidemiology in Filarial Nematodes: Transforming the Basis for Elimination Program Decisions. Front Genet, 10, 1282. doi:10.3389/fgene.2019.01282

Hetzel, M. W., Choudhury, A. A., Pulford, J., Ura, Y., Whittaker, M., Siba, P. M., \& Mueller, I. (2014). Progress in mosquito net coverage in Papua New Guinea. Malar J, 13, 242. doi:10.1186/1475-2875-13-242

Hetzel, M. W., Gideon, G., Lote, N., Makita, L., Siba, P. M., \& Mueller, I. (2012). Ownership and usage of mosquito nets after four years of large-scale free distribution in Papua New Guinea. Malar J, 11(1), 192. doi:10.1186/1475-2875-11-192

Hetzel, M. W., Reimer, L. J., Gideon, G., Koimbu, G., Barnadas, C., Makita, L., . . Mueller, I. (2016). Changes in malaria burden and transmission in sentinel sites after the roll-out of long-lasting insecticidal nets in Papua New Guinea. Parasit Vectors, 9(1), 340. doi:10.1186/s13071-016$1635-x$

Hii, J. L., Smith, T., Vounatsou, P., Alexander, N., Mai, A., Ibam, E., \& Alpers, M. P. (2001). Area effects of bednet use in a malaria-endemic area in Papua New Guinea. Trans $R$ Soc Trop Med Hyg, 95(1), 7-13. doi:10.1016/s0035-9203(01)90315-3

Hofmann, N. E., Karl, S., Wampfler, R., Kiniboro, B., Teliki, A., Iga, J., ... Mueller, I. (2017). The complex relationship of exposure to new Plasmodium infections and incidence of clinical malaria in Papua New Guinea. Elife, 6. doi:10.7554/eLife.23708

This article is protected by copyright. All rights reserved 
Hong, N. V., Delgado-Ratto, C., Thanh, P. V., Van den Eede, P., Guetens, P., Binh, N. T., ... RosanasUrgell, A. (2016). Population Genetics of Plasmodium vivax in Four Rural Communities in Central Vietnam. PLoS NegI Trop Dis, 10(2), e0004434. doi:10.1371/journal.pntd.0004434 Hupalo, D. N., Luo, Z., Melnikov, A., Sutton, P. L., Rogov, P., Escalante, A., . . Carlton, J. M. (2016). Population genomics studies identify signatures of global dispersal and drug resistance in Plasmodium vivax. Nat Genet, 48(8), 953-958. doi:10.1038/ng.3588

Hurlbert, S. H. (1971). The Nonconcept of Species Diversity: A Critique and Alternative Parameters. Ecology, 52(4), 577-586. doi:10.2307/1934145

Imwong, M., Nair, S., Pukrittayakamee, S., Sudimack, D., Williams, J. T., Mayxay, M., . . Anderson, T. J. (2007). Contrasting genetic structure in Plasmodium vivax populations from Asia and South America. Int J Parasitol, 37(8-9), 1013-1022. doi:10.1016/j.ijpara.2007.02.010

Imwong, M., Sudimack, D., Pukrittayakamee, S., Osorio, L., Carlton, J. M., Day, N. P., . . Anderson, T. J. (2006). Microsatellite variation, repeat array length, and population history of Plasmodium vivax. Mol Biol Evol, 23(5), 1016-1018. doi:10.1093/molbev/msj116

Iwagami, M., Fukumoto, M., Hwang, S. Y., Kim, S. H., Kho, W. G., \& Kano, S. (2012). Population structure and transmission dynamics of Plasmodium vivax in the Republic of Korea based on microsatellite DNA analysis. PLoS Negl Trop Dis, 6(4), e1592. doi:10.1371/journal.pntd.0001592

Jakobsson, M., \& Rosenberg, N. A. (2007). CLUMPP: a cluster matching and permutation program for dealing with label switching and multimodality in analysis of population structure. Bioinformatics, 23(14), 1801-1806. doi:10.1093/bioinformatics/btm233

Jennison, C., Arnott, A., Tessier, N., Tavul, L., Koepfli, C., Felger, I., . . Barry, A. E. (2015). Plasmodium vivax populations are more genetically diverse and less structured than sympatric Plasmodium falciparum populations. PLoS Negl Trop Dis, 9(4), e0003634. doi:10.1371/journal.pntd.0003634

Jost, L. (2008). G(ST) and its relatives do not measure differentiation. Mol Ecol, 17(18), 4015-4026. doi:10.1111/j.1365-294x.2008.03887.x

Karunaweera, N. D., Ferreira, M. U., Hartl, D. L., \& Wirth, D. F. (2006). Fourteen polymorphic microsatellite DNA markers for the human malaria parasite Plasmodium vivax. Molecular Ecology Notes, 7(1), 172-175. doi:10.1111/j.1471-8286.2006.01534.x

Kattenberg, J. H., Gumal, D. L., Ome-Kaius, M., Kiniboro, B., Philip, M., Jally, S., . . Robinson, L. J. (2020). The epidemiology of Plasmodium falciparum and Plasmodium vivax in East Sepik Province, Papua New Guinea, pre- and post-implementation of national malaria control efforts Accepted for publication in Malaria Journal. 
Keenan, K., McGinnity, P., Cross, T. F., Crozier, W. W., \& Prodöhl, P. A. (2013). diveRsity: An R package for the estimation and exploration of population genetics parameters and their associated errors. Methods in Ecology and Evolution, 4(8), 782-788. doi:10.1111/2041-210X.12067

Koepfli, C., \& Mueller, I. (2017). Malaria Epidemiology at the Clone Level. Trends Parasitol, 33(12), 974-985.

Koepfli, C., Ome-Kaius, M., Jally, S., Malau, E., Maripal, S., Ginny, J., . . Robinson, L. J. (2017). Sustained malaria control over an eight-year period in Papua New Guinea: the challenge of low-density asymptomatic infections. J Infect Dis. doi:10.1093/infdis/jix507

Koepfli, C., Robinson, L. J., Rarau, P., Salib, M., Sambale, N., Wampfler, R., . . Mueller, I. (2015). Blood-Stage Parasitaemia and Age Determine Plasmodium falciparum and P. vivax Gametocytaemia in Papua New Guinea. PLoS One, 10(5), e0126747. doi:10.1371/journal.pone.0126747

Koepfli, C., Timinao, L., Antao, T., Barry, A. E., Siba, P., Mueller, I., \& Felger, I. (2013). A Large Plasmodium vivax Reservoir and Little Population Structure in the South Pacific. PLoS One, 8(6), e66041. doi:10.1371/journal.pone.0066041

Koleala, T., Karl, S., Laman, M., Moore, B. R., Benjamin, J., Barnadas, C., ... Davis, T. M. (2015). Temporal changes in Plasmodium falciparum anti-malarial drug sensitivity in vitro and resistance-associated genetic mutations in isolates from Papua New Guinea. Malar J, 14, 37. doi:10.1186/s12936-015-0560-3

Lin, E., Kiniboro, B., Gray, L., Dobbie, S., Robinson, L., Laumaea, A., . . Mueller, I. (2010). Differential patterns of infection and disease with P. falciparum and P. vivax in young Papua New Guinean children. PLoS One, 5(2), e9047. doi:10.1371/journal.pone.0009047

Lischer, H. E. L., \& Excoffier, L. (2012). PGDSpider: an automated data conversion tool for connecting population genetics and genomics programs. Bioinformatics, 28(2), 298-299. doi:10.1093/bioinformatics/btr642

Liu, W., Li, Y., Shaw, K. S., Learn, G. H., Plenderleith, L. J., Malenke, J. A., ... Sharp, P. M. (2014). African origin of the malaria parasite Plasmodium vivax. Nat Commun, 5, 3346. doi:10.1038/ncomms4346

Loy, D. E., Liu, W., Li, Y., Learn, G. H., Plenderleith, L. J., Sundararaman, S. A., .. Hahn, B. H. (2017). Out of Africa: origins and evolution of the human malaria parasites Plasmodium falciparum and Plasmodium vivax. International Journal for Parasitology, 47(2), 87-97. doi:https://doi.org/10.1016/j.ijpara.2016.05.008

Luikart, G., Allendorf, F. W., Cornuet, J. M., \& Sherwin, W. B. (1998). Distortion of allele frequency distributions provides a test for recent population bottlenecks. J Hered, 89(3), 238-247. 
malEra Consultative Group on Monitoring, E., \& Surveillance. (2011). A research agenda for malaria eradication: monitoring, evaluation, and surveillance. PLoS Med, 8(1), e1000400. doi:10.1371/journal.pmed.1000400

Matschiner, M., \& Salzburger, W. (2009). TANDEM: integrating automated allele binning into genetics and genomics workflows. Bioinformatics, 25(15), 1982-1983. doi:10.1093/bioinformatics/btp303

McNamara, D. T., Kasehagen, L. J., Grimberg, B. T., Cole-Tobian, J., Collins, W. E., \& Zimmerman, P. A. (2006). Diagnosing infection levels of four human malaria parasite species by a polymerase chain reaction/ligase detection reaction fluorescent microsphere-based assay. Am J Trop Med Hyg, 74(3), 413-421.

Messerli, C., Hofmann, N. E., Beck, H. P., \& Felger, I. (2017). Critical Evaluation of Molecular Monitoring in Malaria Drug Efficacy Trials and Pitfalls of Length-Polymorphic Markers. Antimicrob Agents Chemother, 61(1). doi:10.1128/AAC.01500-16

Miotto, O., Almagro-Garcia, J., Manske, M., Macinnis, B., Campino, S., Rockett, K. A., . . Kwiatkowski, D. P. (2013). Multiple populations of artemisinin-resistant Plasmodium falciparum in Cambodia. Nat Genet, 45(6), 648-655. doi:10.1038/ng.2624

Mita, T., Hombhanje, F., Takahashi, N., Sekihara, M., Yamauchi, M., Tsukahara, T., . . Ohashi, J. (2018). Rapid selection of sulphadoxine-resistant Plasmodium falciparum and its effect on within-population genetic diversity in Papua New Guinea. Scientific Reports, 8(1), 5565. doi:10.1038/s41598-018-23811-7

Mu, J., Awadalla, P., Duan, J., McGee, K. M., Joy, D. A., McVean, G. A., \& Su, X. Z. (2005). Recombination hotspots and population structure in Plasmodium falciparum. PLOS Biol, 3(10), e335. doi:10.1371/journal.pbio.0030335

Mueller, I., Bockarie, M., Alpers, M., \& Smith, T. (2003). The epidemiology of malaria in Papua New Guinea. Trends Parasitol, 19(6), 253-259.

Mueller, I., Widmer, S., Michel, D., Maraga, S., McNamara, D. T., Kiniboro, B., . . Zimmerman, P. A. (2009). High sensitivity detection of Plasmodium species reveals positive correlations between infections of different species, shifts in age distribution and reduced local variation in Papua New Guinea. Malar J, 8, 41. doi:10.1186/1475-2875-8-41

Neafsey, D. E., Galinsky, K., Jiang, R. H., Young, L., Sykes, S. M., Saif, S., . . Carlton, J. M. (2012). The malaria parasite Plasmodium vivax exhibits greater genetic diversity than Plasmodium falciparum. Nat Genet, 44(9), 1046-1050. doi:10.1038/ng.2373

Nei, M. (1987). Molecular Evolutionary Genetics: Columbia University Press. 
Nkhoma, S. C., Nair, S., Al-Saai, S., Ashley, E., McGready, R., Phyo, A. P., . . Anderson, T. J. (2013). Population genetic correlates of declining transmission in a human pathogen. Mol Ecol, 22(2), 273-285. doi:10.1111/mec.12099

Noviyanti, R., Coutrier, F., Utami, R. A., Trimarsanto, H., Tirta, Y. K., Trianty, L., . . Auburn, S. (2015). Contrasting Transmission Dynamics of Co-endemic Plasmodium vivax and P. falciparum: Implications for Malaria Control and Elimination. PLoS Neg/ Trop Dis, 9(5), e0003739. doi:10.1371/journal.pntd.0003739

Oliveira-Ferreira, J., Lacerda, M. V., Brasil, P., Ladislau, J. L., Tauil, P. L., \& Daniel-Ribeiro, C. T. (2010). Malaria in Brazil: an overview. Malar J, 9, 115. doi:10.1186/1475-2875-9-115

Olliaro, P. L., Barnwell, J. W., Barry, A., Mendis, K., Mueller, I., Reeder, J. C., .. W Wongsrichanalai, C. (2016). Implications of Plasmodium vivax Biology for Control, Elimination, and Research. Am J Trop Med Hyg, 95(6 Suppl), 4-14. doi:10.4269/ajtmh.16-0160

Orjuela-Sanchez, P., Da Silva-Nunes, M., Da Silva, N. S., Scopel, K. K., Goncalves, R. M., Malafronte, R. S., \& Ferreira, M. U. (2009). Population dynamics of genetically diverse Plasmodium falciparum lineages: community-based prospective study in rural Amazonia. Parasitology, 136(10), 1097-1105. doi:10.1017/S0031182009990539

Orjuela-Sanchez, P., Sa, J. M., Brandi, M. C., Rodrigues, P. T., Bastos, M. S., Amaratunga, C., . . Ferreira, M. U. (2013). Higher microsatellite diversity in Plasmodium vivax than in sympatric Plasmodium falciparum populations in Pursat, Western Cambodia. Exp Parasitol, 134(3), 318326. doi:10.1016/j.exppara.2013.03.029

Paul, R. E., Packer, M. J., Walmsley, M., Lagog, M., Ranford-Cartwright, L. C., Paru, R., \& Day, K. P. (1995). Mating patterns in malaria parasite populations of Papua New Guinea. Science, 269(5231), 1709-1711.

Pava, Z., Noviyanti, R., Handayuni, I., Trimarsanto, H., Trianty, L., Burdam, F. H., . . Auburn, S. (2017). Genetic micro-epidemiology of malaria in Papua Indonesia: Extensive P. vivax diversity and a distinct subpopulation of asymptomatic P. falciparum infections. PLoS One, 12(5), e0177445. doi:10.1371/journal.pone.0177445

Pearson, R. D., Amato, R., Auburn, S., Miotto, O., Almagro-Garcia, J., Amaratunga, C., . . . Kwiatkowski, D. P. (2016). Genomic analysis of local variation and recent evolution in Plasmodium vivax. Nat Genet, 48(8), 959-964. doi:10.1038/ng.3599

Piry, S., Luikart, G., \& Cornuet, J. BOTTLENECK : A program for detecting recent effective population size reductions from allele data frequencies. Retrieved from http://www1.montpellier.inra.fr/CBGP/software/Bottleneck/pub.html 
Pritchard, J. K., Stephens, M., \& Donnelly, P. (2000). Inference of population structure using multilocus genotype data. Genetics, 155(2), 945-959.

Riley, I. D. (1983). Population change and distribution in Papua New Guinea: an epidemiological approach. Journal of Human Evolution, 12(1), 125-132. doi:https://doi.org/10.1016/S00472484(83)80017-7

Robinson, L. J., Wampfler, R., Betuela, I., Karl, S., White, M. T., Li Wai Suen, C. S., . . Mueller, I. (2015). Strategies for understanding and reducing the Plasmodium vivax and Plasmodium ovale hypnozoite reservoir in Papua New Guinean children: a randomised placebo-controlled trial and mathematical model. PLoS Med, 12(10), e1001891. doi:10.1371/journal.pmed.1001891

Rosanas-Urgell, A., Mueller, D., Betuela, I., Barnadas, C., Iga, J., Zimmerman, P. A., . . Felger, I. (2010). Comparison of diagnostic methods for the detection and quantification of the four sympatric Plasmodium species in field samples from Papua New Guinea. Malar J, 9, 361. doi:10.1186/1475-2875-9-361

Rosenberg, N. A. (2004). distruct: a program for the graphical display of population structure. Molecular Ecology Notes, 4(1), 137-138. doi:10.1046/j.1471-8286.2003.00566.x

Salgueiro, P., Vicente, J. L., Figueiredo, R. C., \& Pinto, J. (2016). Genetic diversity and population structure of Plasmodium falciparum over space and time in an African archipelago. Infect Genet Evol, 43, 252-260. doi:10.1016/j.meegid.2016.06.001

Schultz, L., Wapling, J., Mueller, I., Ntsuke, P., Senn, N., Nale, J., . . Barry, A. (2010). Multilocus haplotypes reveal variable levels of diversity and population structure of Plasmodium falciparum in Papua New Guinea, a region of intense perennial transmission. Malaria Journal, 9(1), 336.

Senn, N., Rarau, P., Stanisic, D. I., Robinson, L., Barnadas, C., Manong, D., . . Mueller, I. (2012). Intermittent Preventive Treatment for Malaria in Papua New Guinean Infants Exposed to Plasmodium falciparum and P. vivax: A Randomized Controlled Trial. PLOS Medicine, 9(3), e1001195. doi:10.1371/journal.pmed.1001195

Smith, J. M., Smith, N. H., O'Rourke, M., \& Spratt, B. G. (1993). How clonal are bacteria? Proceedings of the National Academy of Sciences, 90(10), 4384-4388. doi:10.1073/pnas.90.10.4384

Susomboon, P., Iwagami, M., Tangpukdee, N., Krusood, S., Looareesuwan, S., \& Kano, S. (2008). Differences in genetic population structures of Plasmodium falciparum isolates from patients along Thai-Myanmar border with severe or uncomplicated malaria. Malar J, 7, 212. doi:10.1186/1475-2875-7-212

This article is protected by copyright. All rights reserved 
Sutton, P. L. (2013). A call to arms: on refining Plasmodium vivax microsatellite marker panels for comparing global diversity. Malar J, 12, 447. doi:10.1186/1475-2875-12-447

Tessema, S. K., Monk, S. L., Schultz, M. B., Tavul, L., Reeder, J. C., Siba, P. M., . . Barry, A. E. (2015). Phylogeography of var gene repertoires reveals fine-scale geospatial clustering of Plasmodium falciparum populations in a highly endemic area. Mol Ecol, 24(2), 484-497. doi:10.1111/mec.13033

Vardo-Zalik, A. M., Zhou, G., Zhong, D., Afrane, Y. A., Githeko, A. K., \& Yan, G. (2013). Alterations in Plasmodium falciparum genetic structure two years after increased malaria control efforts in western Kenya. Am J Trop Med Hyg, 88(1), 29-36. doi:10.4269/ajtmh.2012.12-0308

Volkman, S. K., Sabeti, P. C., DeCaprio, D., Neafsey, D. E., Schaffner, S. F., Milner, D. A., Jr., . . Wirth, D. F. (2007). A genome-wide map of diversity in Plasmodium falciparum. Nat Genet, 39(1), 113-119. doi:10.1038/ng1930

Wahlund, S. (1928). Zusammensetzung Von Populationen Und Korrelationserscheinungen Vom Standpunkt Der Vererbungslehre Aus Betrachtet. Hereditas, 11(1), 65--106. doi:10.1111/j.1601-5223.1928.tb02483.x

Waltmann, A., Darcy, A. W., Harris, I., Koepfli, C., Lodo, J., Vahi, V., . . Mueller, I. (2015). High Rates of Asymptomatic, Sub-microscopic Plasmodium vivax Infection and Disappearing Plasmodium falciparum Malaria in an Area of Low Transmission in Solomon Islands. PLoS Neg/Trop Dis, 9(5), e0003758. doi:10.1371/journal.pntd.0003758

Waltmann, A., Koepfli, C., Tessier, N., Karl, S., Fola, A., Darcy, A. W., .. Barry, A. E. (2018). Increasingly inbred and fragmented populations of Plasmodium vivax associated with the eastward decline in malaria transmission across the Southwest Pacific. PLoS Negl Trop Dis, 12(1), e0006146.

Weir, B. S., \& Cockerham, C. C. (1984). Estimating F-Statistics for the Analysis of Population Structure. Evolution, 38(6), 1358-1370. doi:10.2307/2408641

White, N. J. (2011). Determinants of relapse periodicity in Plasmodium vivax malaria. Malar J, 10, 297. doi:10.1186/1475-2875-10-297

WHO. (2017). World Malaria Report 2017. Retrieved from Geneva:

WHO. (2018). World Malaria Report 2018. Retrieved from Geneva: http://www.who.int/malaria/publications/world-malaria-report-2015/report/en/ WHO. (2019). World Malaria Report 2019. Retrieved from Geneva:

Wlodarska, M., Johnston, J. C., Gardy, J. L., \& Tang, P. (2015). A microbiological revolution meets an ancient disease: improving the management of tuberculosis with genomics. Clinical Microbiology Reviews, 28(2), 523-539. doi:10.1128/CMR.00124-14

This article is protected by copyright. All rights reserved 
Data accessibility

992

1. Microsatellite genotypes

were

deposited

in

Dryad:

https://doi.org/10.5061/dryad.37pvmcvfh.

994

995

\section{Author Contributions}

996

AEB, IM and JK identified the research problem and designed the research project, MO-K, PS, IF, M, JK and LJR co-ordinated the field studies and molecular epidemiology, CK, DL-N, CB processed samples and performed molecular assays, JHK, ZR, RK and AAF conducted the microsatellite genotyping and analysed data, CJ and CK contributed data, JHK and AEB

1000 wrote the paper.

1001

1002

1003

Figure Legends

1004

Figure 1. Map of the study areas and infection prevalence from 2005-2016. (A) Map of East Sepik and Madang study area villages and locations on the north coast of Papua New Guinea (inset) The graphs show the pre-LLIN (2005/6) and post-LLIN (2010-2014) molecular prevalence for (B) East Sepik and (C) Madang for both $P$. falciparum (light grey) and $P$. vivax (dark grey) and proportion of multiclonal infections (black line) (Arnott et al., 2013; Barry et al., 2013; Kattenberg et al., 2020; Koepfli et al., 2017; Koepfli et al., 2015; Mueller et al., 2009; Senn et al., 2012).

1011

Figure 2. Changing diversity of $\boldsymbol{P}$. falciparum and $\boldsymbol{P}$. vivax populations over an

1012 intensifying period of malaria control (2005-2014). Allelic Richness (Rs) in P. falciparum $(A)(n=860)$ and $P$. vivax $(B)(n=755)$ populations pre- $(\leq 2006)$ and post-LLIN $(\geq 2010)$ massdistributions. Error bars indicate standard deviations.

\section{Figure 3. Genetic differentiation estimates among $P$. falciparum and $P$. vivax} populations pre- and post-LLIN mass-distributions. Pairwise Jost's $D$ values for (A) $P$. falciparum and (B) P. vivax. Pairwise Jost's $D$ values and $95 \%$ confidence intervals were estimated with 1000 bootstraps using the diveRsity package in R. Pairwise $F_{S T}$ values (Weir and Cockerham) are shown in figure S1. 
1020 Figure 4. Bayesian cluster analysis of $\boldsymbol{P}$. falciparum (A) and $\boldsymbol{P}$. vivax (B) of pre- (2005/6)

1021 and post-LLIN studies (2010-2014). Individual samples are sorted by province and year 1022 (solid lines), catchment area (dashed line) and cluster membership (colour). Madang 1023 catchments are organised as Malala, Mugil, Utu and ESP2 and 2012-13 as Brukham, Burui, 1024 Ilahita, Ulupu, and Wombisa (no infections in 2012). As identified in the genetic differentiation 1025 analysis (Jost's D, Figure 3), there was moderate differentiation for $P$. falciparum between 1026 the ESP1 (Wosera area) 2005 and Madang 2006 versus the other studies (only one province 1027 included pre-LLIN), which is believed to be for a large part caused by experimental- and data 1028 analysis differences. Therefore, these $P$. falciparum studies were grouped separately for the 1029 population structure analysis, in order to avoid artificial changes in ancestry over time.

1030 Figure 5. Unrooted neighbour-joining tree of $\boldsymbol{P}$. vivax isolates in East Sepik province in 1031 2012-13. Relatedness among haplotypes was defined by calculating the pairwise distance 1032 and neighbour-joining tree using the APE package in R and the tree was visualized using 1033 Phylocanvas through microreact.org (Argimon et al., 2016; "Microreact,"). Colours and 1034 shapes indicate the village where the isolates were collected. Very closely related isolates 1035 are observed in the village of Sunuhu (dark grey circles).

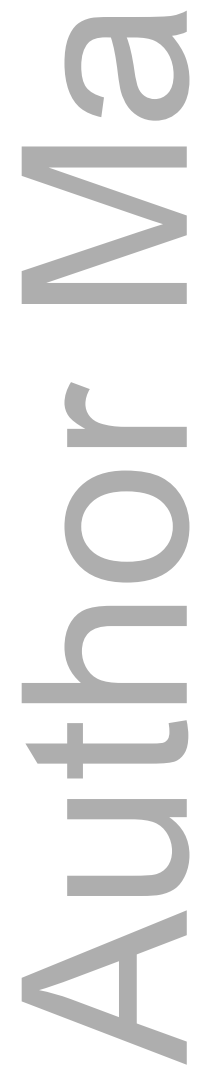


1037 Table 1. Estimates of multi-locus linkage disequilibrium (mLD) for Plasmodium 1038 populations pre- and post-LLIN mass-distribution. $I_{A} s$ is the standardized index of 1039 association; $\mathrm{n}=$ number of complete haplotypes. Single clone infection $\mathrm{mLD}$ is shown in 1040 Table S2.

\begin{tabular}{|c|c|c|c|c|c|c|}
\hline 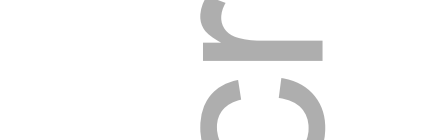 & \multicolumn{3}{|c|}{$\begin{array}{c}\text { All } \\
\text { haplotypes }\end{array}$} & \multicolumn{3}{|c|}{$\begin{array}{c}\text { Unique } \\
\text { haplotypes }\end{array}$} \\
\hline P. falciparu & $\mathbf{n}$ & $I_{A} s$ & $p$-value & $\mathbf{n}$ & $I_{A} \mathrm{~s}$ & p-value \\
\hline all pre-LLIN studies & 199 & 0.0051 & 0.036 & 196 & 0.0048 & 0.046 \\
\hline all post-LLIN studies & 136 & 0.0075 & 0.020 & 116 & -0.003 & 0.787 \\
\hline East Sepik I 2005 & 15 & -0.0038 & 0.566 & 15 & -0.0038 & 0.566 \\
\hline East Sepik II 2005 & 103 & 0.0004 & 0.446 & 100 & -0.0003 & 0.517 \\
\hline East Sepik 2012-13 & 21 & 0.0454 & 0.002 & 19 & 0.0202 & 0.088 \\
\hline Madang & 81 & 0.0108 & 0.011 & 81 & 0.0108 & 0.011 \\
\hline Madang & 82 & 0.0113 & 0.021 & 72 & -0.0046 & 0.802 \\
\hline Madang $\quad 2014$ & 33 & 0.0725 & $<0.00001$ & 25 & 0.0198 & 0.059 \\
\hline All studies & 335 & 0.0044 & 0.021 & 312 & 0.0027 & 0.103 \\
\hline \multicolumn{7}{|l|}{ P. vivax } \\
\hline all pre-LLIN studies & 179 & -0.0002 & 0.540 & 179 & -0.0002 & 0.539 \\
\hline all post-LLIN studies & 125 & 0.0126 & $<0.00001$ & 120 & 0.007 & 0.001 \\
\hline East Sepik I 2005 & 48 & 0.0066 & 0.099 & 48 & 0.0066 & 0.099 \\
\hline East Sepik II 2005 & 37 & 0.0064 & 0.149 & 37 & 0.0064 & 0.149 \\
\hline East Sepik 2012-13 & 20 & 0.2154 & $<0.00001$ & 19 & 0.1981 & $<0.00001$ \\
\hline Madang 2006 & 94 & -0.0022 & 0.796 & 94 & -0.0022 & 0.796 \\
\hline Madang & 80 & 0.0113 & 0.0005 & 77 & 0.0004 & 0.436 \\
\hline Madang & 24 & 0.0073 & 0.209 & 24 & 0.0073 & 0.209 \\
\hline All studies & 303 & 0.0025 & 0.013 & 299 & 0.0017 & 0.057 \\
\hline
\end{tabular}




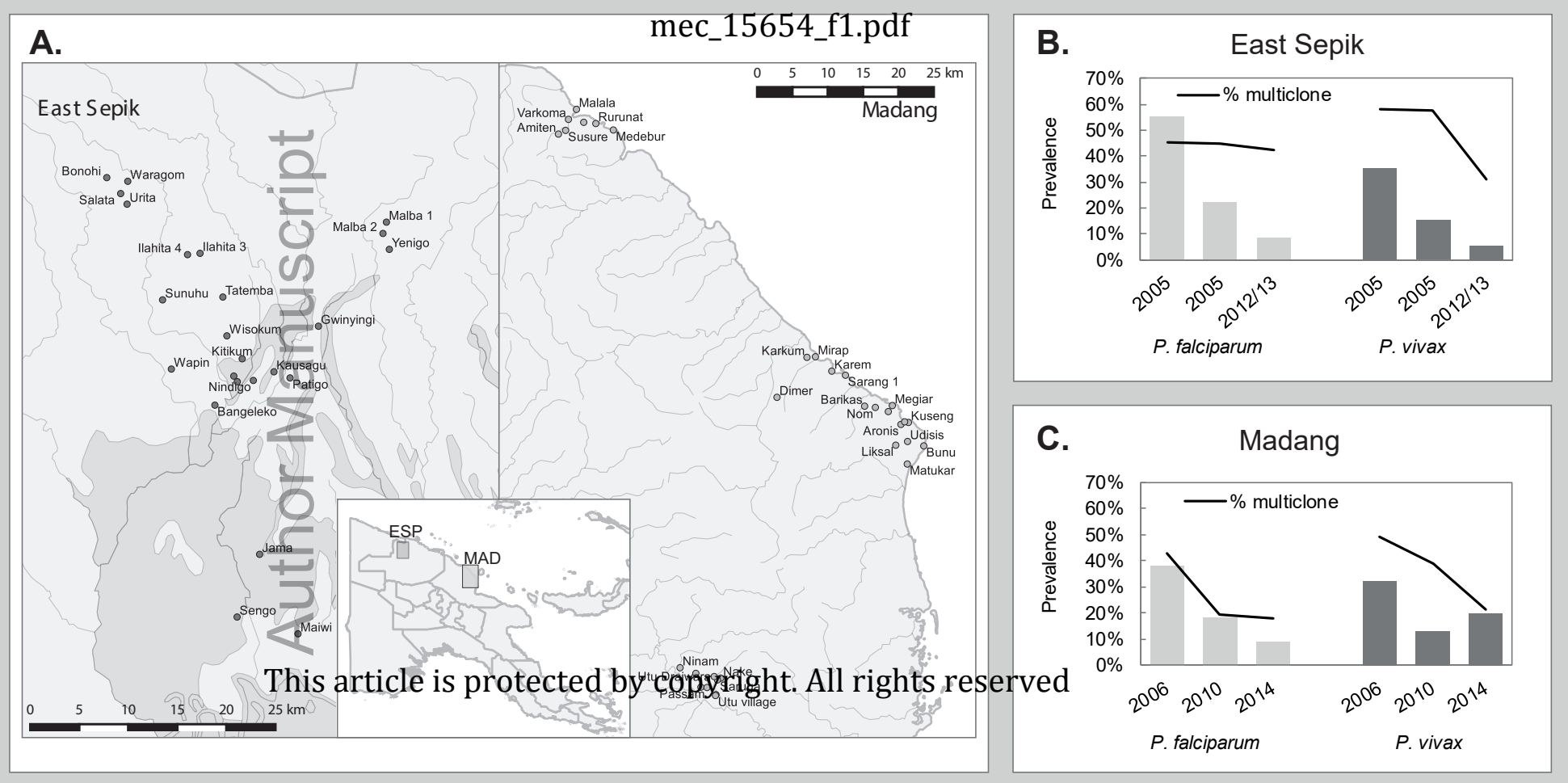




\section{A. $P$, falciparum}

pre-LLIN post-LLIN

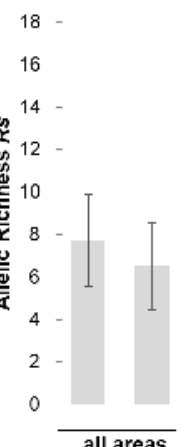

all areas

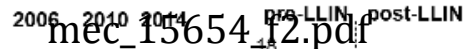

2005

2012

200620102014
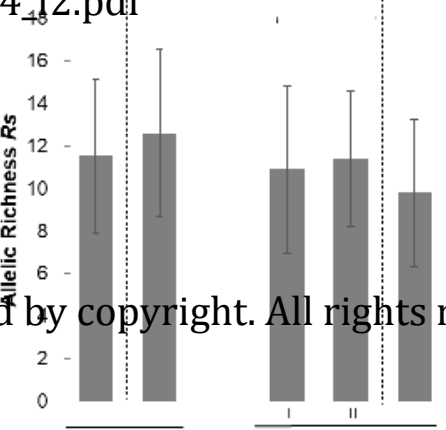

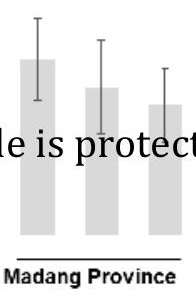

East Sepik Province

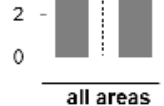

Madang Province
This article is protecteđ̛ by copyright. All rights reserved

East Sepik Province

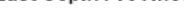




\section{A. P. falciparum}

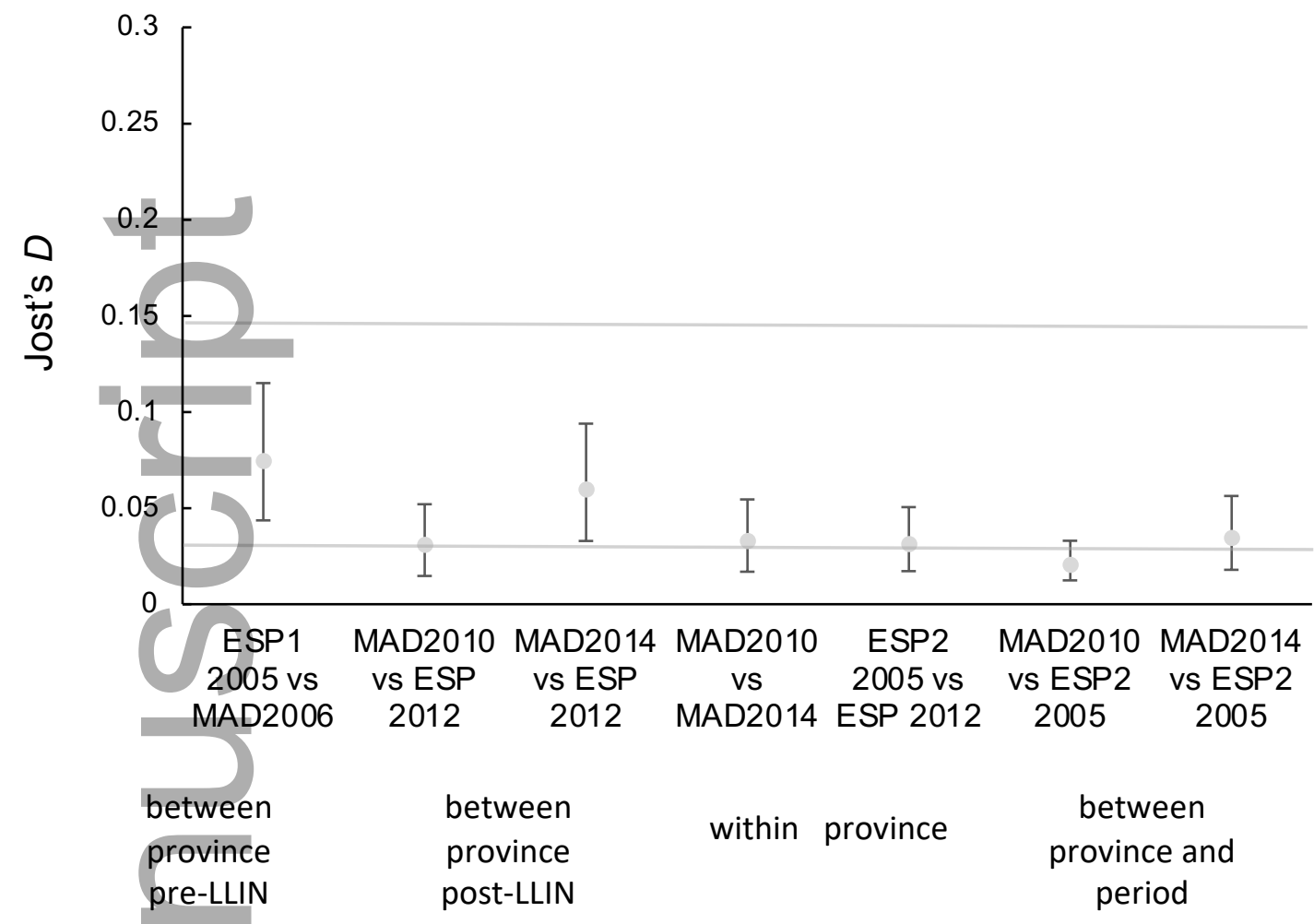

\section{B. $P$. vivax}

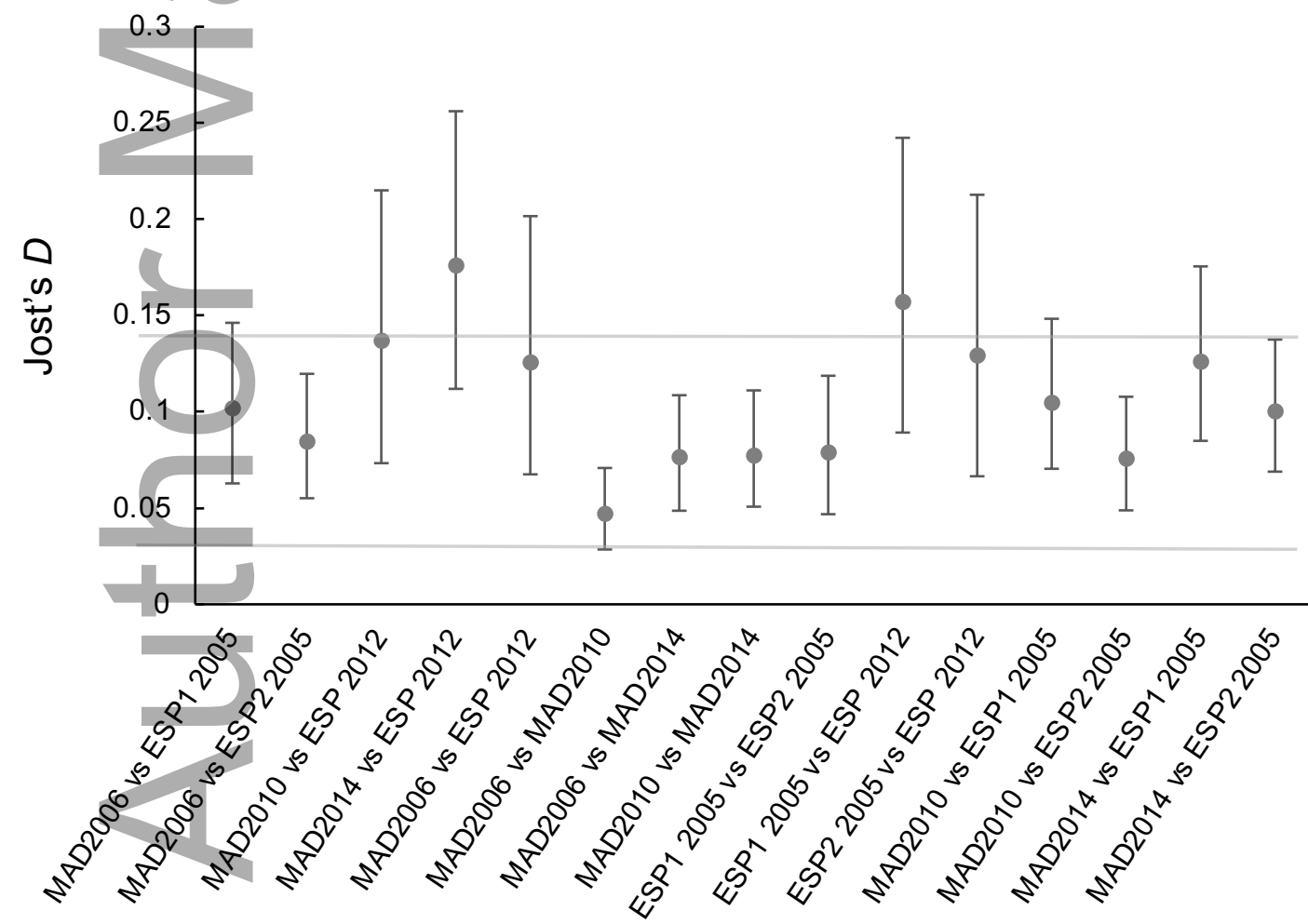

between between

province province

pre-LLIN post-LLIN between

province and

period

This article is protected by copyright. All rights reserved 

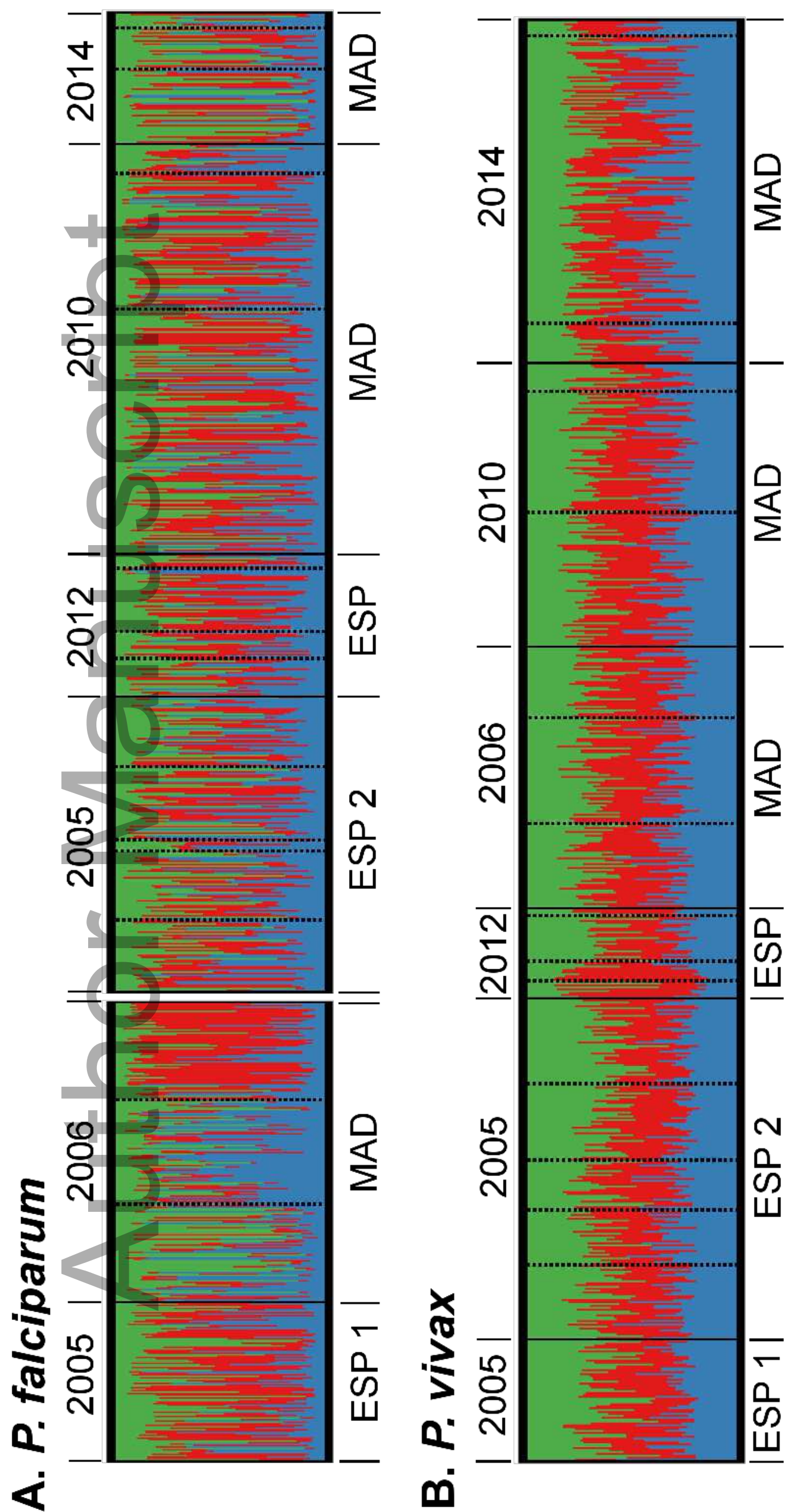

This article is protected by copyright. All rights reserved 
Illhaita_3 Ilhaita_4

$\triangle$ Jama

A Maiwi

$\square$ Malba_1

Malba_2

Salata

$\Delta$ Sengo

Sunuhu

岤 Urita

Waragom 


\section{University Library}

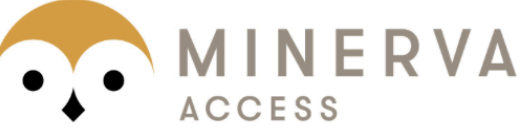

A gateway to Melbourne's research publications

Minerva Access is the Institutional Repository of The University of Melbourne

\section{Author/s:}

Kattenberg, JH;Razook, Z;Keo, R;Koepfli, C;Jennison, C;Lautu-Gumal, D;Fola, AA;OmeKaius, M;Barnadas, C;Siba, P;Felger, I;Kazura, J;Mueller, I;Robinson, LJ;Barry, AE

Title:

MonitoringPlasmodium falciparumandPlasmodium vivaxusing microsatellite markers indicates limited changes in population structure after substantial transmission decline in Papua New Guinea

\section{Date:}

2020-10-16

\section{Citation:}

Kattenberg, J. H., Razook, Z., Keo, R., Koepfli, C., Jennison, C., Lautu-Gumal, D., Fola, A. A., Ome-Kaius, M., Barnadas, C., Siba, P., Felger, I., Kazura, J., Mueller, I., Robinson, L. J. \& Barry, A. E. (2020). MonitoringPlasmodium falciparumandPlasmodium vivaxusing microsatellite markers indicates limited changes in population structure after substantial transmission decline in Papua New Guinea. MOLECULAR ECOLOGY, 29 (23), pp.4525-4541. https://doi.org/10.1111/mec. 15654.

Persistent Link:

http://hdl.handle.net/11343/276447 